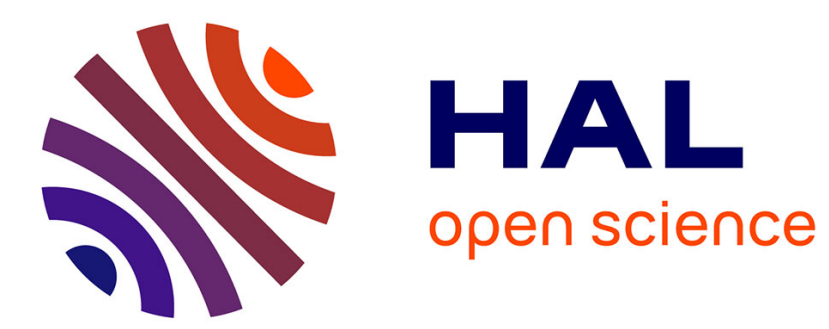

\title{
The contribution of jumps to forecasting the density of returns
}

\author{
Christophe Chorro, Florian Ielpo, Benoît Sévi
}

\section{To cite this version:}

Christophe Chorro, Florian Ielpo, Benoît Sévi. The contribution of jumps to forecasting the density of returns. 2017. halshs-01442618

\section{HAL Id: halshs-01442618 \\ https://shs.hal.science/halshs-01442618}

Submitted on 20 Jan 2017

HAL is a multi-disciplinary open access archive for the deposit and dissemination of scientific research documents, whether they are published or not. The documents may come from teaching and research institutions in France or abroad, or from public or private research centers.
L'archive ouverte pluridisciplinaire HAL, est destinée au dépôt et à la diffusion de documents scientifiques de niveau recherche, publiés ou non, émanant des établissements d'enseignement et de recherche français ou étrangers, des laboratoires publics ou privés. 


\section{Documents de Travail du
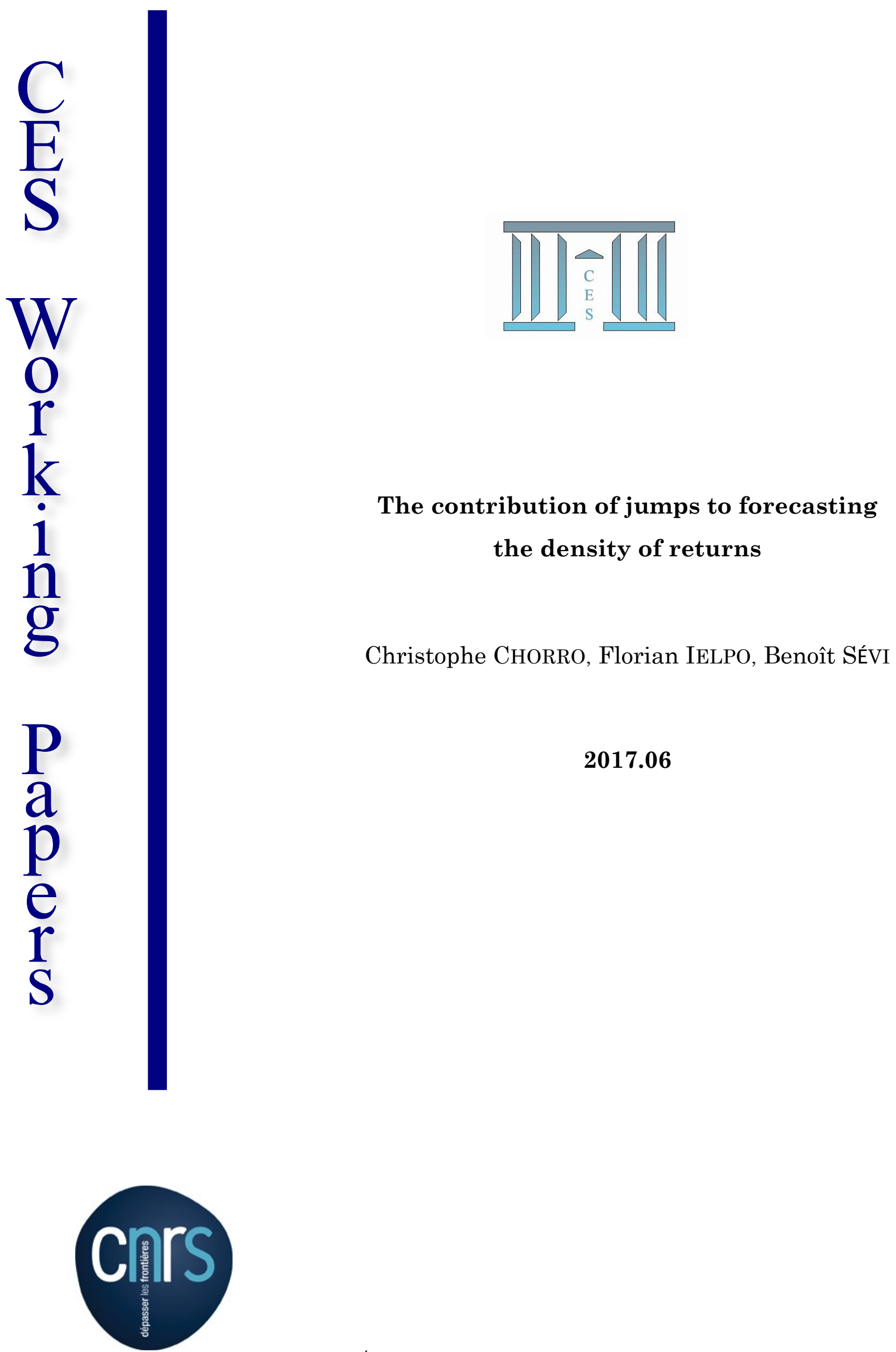


\title{
The contribution of jumps to forecasting the density of returns *
}

\author{
Christophe Chorro $^{\dagger}$ \\ Paris 1 Sorbonne University and Labex ReFi \\ Florian Ielpo $\ddagger$ \\ Unigestion SA, Paris 1 Sorbonne University, Labex ReFi and IPAG \\ Benoît Sévi ${ }^{\S}$ \\ Nantes University
}

January 12, 2017

\begin{abstract}
The extraction of the jump component in the dynamics of asset prices has witnessed a considerably growing body of literature. Of particular interest is the decomposition of returns' quadratic variation between their continuous and jump components. Recent contributions highlight the importance of this component in forecasting volatility at different horizons. In this article, we extend a methodology developed in Maheu and McCurdy (2011) to exploit the information content of intraday data in forecasting the density of returns at horizons up to sixty days. We follow Boudt et al. (2011) to detect intraday returns that should be considered as jumps. The methodology is robust to intra-week periodicity and further delivers estimates of signed jumps in contrast to the rest of the literature where only the squared jump component can be estimated. Then, we estimate a bivariate model of returns and volatilities where the jump component is independently modeled using a jump distribution that fits the stylized facts of the estimated jumps. Our empirical results for S\&P 500 futures, U.S. 10-year Treasury futures, USD/CAD exchange rate and WTI crude oil futures highlight the importance of considering the continuous/jump decomposition for density forecasting while this is not the case for volatility point forecast. In particular, we show that the model considering jumps apart from the continuous component consistently deliver better density forecasts for forecasting horizons ranging from 1 to 30 days.
\end{abstract}

JEL Classification: C15, C32, C53, G1

Keywords: density forecasting, jumps, realized volatility, bipower variation, median realized volatility, leverage effect.

*For the first two authors, this work was achieved through the Laboratory of Excellence on Financial Regulation (Labex ReFi) supported by PRES heSam under the reference ANR10LABX0095. It benefited from a French government support managed by the National Research Agency (ANR) within the project Investissements d'Avenir Paris Nouveaux Mondes (investments for the future Paris New Worlds) under the reference ANR11IDEX000602. Part of the paper was written while the third author was visiting researcher at the London Business School. The authors are thankful to Derek Bunn, Walter Distaso, Dobrislav Dobrev, Per Frederiksen, Rehim Kilic, Robert Kosowski, Sébastien Laurent, John Maheu, Nour Meddahi, Diaa Noureldin, Petra Posedel, Anders Rahbek, Kevin Sheppard, Stephen Taylor, Almut Veraart and Filip Zikes for many insightful comments. Helpful comments were received from participants at the 2009 Université Paris Ouest Econometrics Workshop in Nanterre, the 2nd Humbolt-Copenhagen Conference on Financial Econometrics in Copenhagen (2011), the 18th International Conference on Forecasting Financial Markets in Marseilles (2011), the Computational and Financial Econometrics Conference (2011) in London and the ESEM meeting in Oslo (2011).

${ }^{\dagger}$ University Paris 1 Panthéon-Sorbonne - CES, Maison des Sciences Économiques, 106-113 Boulevard de l'Hôpital, 75013 Paris, France and Labex Refi. Email: cchorro@univ-paris1.fr.

${ }^{\ddagger}$ Unigestion SA, Avenue de Champel 8C, 1211 Geneva, Switzerland. University Paris 1 Panthéon-Sorbonne - CES, Maison des Sciences Économiques, 106-113 Boulevard de l'Hôpital, 75013 Paris, France. IPAG Business School, Boulevard Saint Germain, 75006 Paris France. Email: fie@unigestion.com.

${ }^{\S}$ LEMNA, BP 52231 Chemin de la Censive du Tertre, 44322 Nantes Cedex, France, Email: benoit.sevi@gmail.com 


\section{Introduction}

When it comes to forecasting the distribution of returns for risk management purposes, is this separation between volatility and jumps equally important? In a reference paper, Andersen et al. (2007a) provide empirical evidence that disentangling jumps from the continuous component significantly help in forecasting the realized volatility at horizons up to 22 days. The explanation for this result is the strong persistence in the continuous component and the absence of autocorrelation in the jump component. Recently, Hansen et al. (2011), Maheu and McCurdy (2011), and Shephard and Sheppard (2010) have suggested "complete" models of returns and volatility. In particular, Maheu and McCurdy (2011) propose a bivariate specification of returns and volatility to obtain density forecasts at horizons up to 60 days. They confirm, in the density context, numerous previous findings that intraday data improve forecasts. We merge these two strands of this recent literature to investigate whether the separation between the continuous and the jump components is of central importance in predicting the density of returns. Our results strongly argue in favor of separating the two components when forecasting the density of returns up to 60 days. Indeed, disentangling jumps from the continuous component help in forecasting the density of returns.

Econometric methods used to disentangle jumps and volatility are unveiling new empirical questions. While recent developments in financial econometrics allow to derive better forecasts of return densities (see Corradi and Swanson (2006) for a recent survey), the issue of the inclusion of the jump component and its information content for such a purpose has not been investigated to date. Hence, in this paper, we examine whether this refinement to use a clear differentiation between jumps and volatility is empirically worth the trouble in a density forecasting exercise. We conduct such an analysis at various horizons (up to 60 days) thus allowing a very detailed analysis of the effects we are interested in.

Forecasting realized volatility has shown to be essential in empirical finance applications such as portfolio choice (Fleming et al. (2003)), risk management activities (Clements et al. (2008)) or derivatives pricing (Christoffersen et al. (2014)). More generally, forecasts of the future values of economic variables are used widely in decision making. Point forecasts, the often traditional focus, are better seen as the central points of ranges of uncertainty. Consequently, to provide a complete description of the uncertainty associated with the point forecast many professional forecasters now publish density forecasts, or more popularly fan charts. In contrast to interval forecasts, which give the probability that the outcome will fall within a stated interval, density forecasts provide a complete description of the uncertainty associated with a forecast. They can thus be seen to provide information on all possible intervals. In conjunction with the increased use of density forecasts by professional forecasters and central banks, the academic literature has also devoted increased emphasis to density forecasting (for surveying methods for predictive density evaluation, see among others Tay and Wallis (2000), Clements (2005), Corradi and Swanson (2006), Timmermann (2006) and Wallis (2007) and the corresponding chapters in Elliott and Timmermann (2016)).

This paper adopts the parsimonious specification of the Heterogeneous Autoregressive Model 
of the Realized Volatility (HAR-RV) model by Corsi (2009) to capture the well-known longmemory dependence in volatility. We also proceed with the detection of jumps following Huang and Tauchen's (2005) statistical test relying on the bipower variation (BPV) estimator. We adapt the test statistic to the more recently developed median realized volatility (MedRV, Andersen et al. (2012)) following the empirical work by Theodossiou and Zikes (2011) and Dumitru and Urga (2012) showing the interesting properties of this estimator.

By using intraday data, it is possible to extract jumps as the difference, when statistically significant, between realized volatility and bipower variation/median realized volatility. This decomposition allows to integrate (or not) jumps for forecasting purposes. Based on the link between the conditional variance and the realized volatility highlighted by Maheu and McCurdy (2011), we estimate different kinds of nested models. Those models are bivariate models based on intraday data including separated jumps (median realized volatility) or not (realized volatility). The motivation behind considering jump-robust measures for realized volatility is that they simply have better predictive properties than non-jump-robust ones (see Shephard and Sheppard (2010), footnote 3).

As mentioned above, we rely on Maheu and McCurdy's (2011) bivariate model to simultaneously estimate returns and volatilities, while assuming a possible leverage effect. The cornerstone of the model is the link that the authors establish between some realized volatility estimators and the "true" conditional volatility in light of the theory underlying these estimators. Such a model allows to derive density forecasts from intraday data for which models comparison can be done. These comparisons between the different bivariate specifications for daily returns and realized volatilities (both "naive" and jump-robust estimators) are conducted using the predictive likelihood tests (Diebold and Mariano, 1995, and Amisano and Giacomini, 2007). We rely on a three nested models: a Gaussian bivariate model using realized variance as well as bivariate models based on median realized variance and a jump component estimated from the dynamics of our pre-estimated intra-day jumps. To these three models we also added two more, using for both realized variance measures and a mixture of two-Gaussian distributions as a conditional distribution, in an effort to assess the quality of the jump detection technique and modeling.

This paper makes two contributions. First, we extend the framework of Maheu and McCurdy (2011) and show how to model jumps in their bivariate framework. Second, we assess the importance of jumps when forecasting the density of returns by comparing jump-robust and non-robust measures of realized volatilities when it comes to forecast the density of returns. Compared to the "naive" measure of realized volatility, considering jumps specifically provides a statistically significant improvement when it comes to forecasting the density of returns, for forecasting horizons ranging from 1 day to 30 days. We thus extend the results in Andersen et al. (2007a) (see also Corsi et al. (2010), Duong and Swanson (2015), Patton and Sheppard (2015)) in showing the importance of disentangling jumps from the continuous component of the distribution of returns for forecasting purpose.

The remainder of the paper is organized as follows. Section 2 summarizes the construction 
of the volatilities and jumps data sets used along with their summary statistics. Section 3 details our modeling and fitting of the estimated jumps' distribution. In Section 4 presents our modeling strategy as well as the methodology used to compare density forecasts. Section 5 discusses the empirical results. Section 6 concludes.

\section{Volatilities and jumps estimation}

This section briefly describes the data and well as the methodology that has been used to estimate jumps and volatilities from four intra-day data sets.

\subsection{Data description}

Our data consist in four futures markets from four different asset classes: stock index, Treasury bond, exchange rate and commodity. While all assets are very liquid (and are therefore suitable for using realized estimators), we had to remove days where the trading activity has not been sufficient to compute these estimators. To this end, we filter our four time series with respect to three parameters: the length of the trading period in the day, the number of zero-returns and the number of transactions. We choose to work with open-to-close returns because overnight returns have shown to follow a very different dynamics. In addition, including overnight returns may alter our analysis when standardizing returns as we work with volatility computed with intraday transaction data. All details about data are relegated in Appendix A.

\section{$2.2 \quad$ Realized quantities}

Let us assume that $p(t)$, the logarithm of the asset price, follows the general stochastic volatility jump diffusion model:

$$
d p(t)=\mu(t) d t+\sigma(t) d W(t)+\kappa(t) d J(t) \quad \text { with } \quad t \geq 0
$$

where the mean $\mu($.$) (predictable drift) is assumed to be continuous and locally bounded, and$ the instantaneous (spot) volatility $\sigma($.$) is strictly positive and càdlàg (right continuous with$ left limit). The mean, as well as the spot volatility, are both assumed to be independent from the driving standard Brownian motion $W(t) .{ }^{1}$ The finite activity counting process, noted $J(t)$, is normalized such that $d J(t)=1$ when a jump occurs at time $t$, and $d J(t)=0$ otherwise. Finally, $\kappa(t)$ is the jump size at time $t$, which is assumed to be random. The process in equation (1), which belongs to the class of Brownian semimartingale processes with jumps, allows returns to exhibit leptokurticity and volatility clustering, which are both relevant empirical characteristics for financial time-series.

If we define $[p](t)$ as the quadratic variation of the process in equation (1), then:

\footnotetext{
${ }^{1}$ The independence assumption is discussed in length in Barndorff-Nielsen and Shephard (2006), who explain that it rules out leverage and volatility feedback effects. The absence of leverage has been recently shown to be empirically relevant for S\&P 500 index and futures returns (see Andersen et al. (2001a), Bollerslev et al. (2006), and Bollerslev et al. (2009) among others).
} 


$$
[p](t)=\operatorname{plim} \sum_{j=0}^{n-1}\left[p\left(\tau_{j+1}-p\left(\tau_{j}\right)\right]^{2}\right.
$$

where $0=\tau_{0}<\tau_{1}<\ldots<\tau_{n}=t$ is a sequence of partitions, and $\sup _{j}\left\{\tau_{j+1}-\tau_{j}\right\} \rightarrow 0$ for $n \rightarrow \infty$. The quadratic variation of process in equation (1) may then be expressed as:

$$
[p](t)=\int_{0}^{t} \sigma^{2}(\tau) d \tau+\sum_{j=1}^{J(t)} \kappa^{2}\left(t_{j}\right)
$$

where $t_{j}$ are times when a jump occurs, implying $d J\left(t_{j}\right)=1$. Eq. (3) represents the continuous sample path along with the jump components of total return variation.

Because most of our analysis will focus on daily returns and volatilities, we consider the daily time interval as unity. For each day $d$, we consider that $M+1$ evenly spaced (calendar time subsampling $^{2}$ ) intraday observations are available for the logarithm of the asset price, noted $p_{d, j}$, thus allowing to compute $M$ continuously compounded intraday returns each day as $r_{d, j}=p_{d, j}-p_{d, j-1}$ for $j=2, \ldots, M$. The realized variance for day $d$ is then given by the sum of squared intraday returns:

$$
R V_{d, M}=\sum_{j=1}^{M} r_{d, j}^{2}
$$

Note that realized variance is the estimator of the total daily variance, and for this reason it remains dependent on the selected sampling frequency $1 / M$. As frequency tends to infinity (if intraday observations are available as often as desired), then:

$$
R V_{d, M} \rightarrow p \int_{d-1}^{d} \sigma^{2}(\tau) d \tau+\sum_{j=J(d-1)}^{J(d)} \kappa^{2}\left(t_{j}\right)
$$

Equation (5) illustrates that volatility, which is by nature a latent variable, is made "observable" by the theory of quadratic variation. Realized volatility for our four assets are plotted in Figures A.1 to A.4 along with price and return series.

The next question addresses how to disentangle jumps from the continuous component. For this purpose, measures of diffusive volatility are necessary. Barndorff-Nielsen and Shephard (2004) tackle this issue by proposing the bipower variation (BPV) measure, which is computed as the scaled summation of the product of adjacent absolute returns. Formally, BPV is defined as follows:

$$
B P V_{d, M}=\xi_{1} \sum_{j=1}^{M-1}\left|r_{d, j}\right|\left|r_{d, j+1}\right|
$$

\footnotetext{
${ }^{2}$ The case of alternative subsampling such as business (or transaction) time subsampling has attracted some attention in the literature (Ané and Geman (2000), Oomen (2006), Andersen et al. (2010)), as it allows to recover normality for standardized returns. Nevertheless, to our best knowledge, these ideas lack asymptotic theory and have not been extended yet to the analysis of jump-robust estimators.
} 
where $\xi_{p} \equiv 2^{p / 2} \Gamma\left(\frac{1 / 2(p+1)}{\Gamma(1 / 2)}\right)=E\left(|Z|_{p}\right)$ denotes the mean of the absolute value of standard normally distributed random variable ${ }^{3}, Z$. The BPV is a consistent estimator of integrated volatility, and allows to decompose the realized volatility into its diffusive and non-diffusive parts. As the sampling frequency increases, the presence of jumps should have no impact, because the return representing the jump is multiplied by a non-jump return which tends to zero asymptotically. This is true in case of rare jumps (one each day), when the probability of two consecutive jumps is negligible.

Nevertheless, the BPV has major drawbacks in empirical applications. First, in practice, the sampling frequency is not high enough to eliminate the influence of jumps. Indeed, the adjacent return does not tend to zero and thus multiply the large (jump) intraday return thus resulting in an upward bias of the BPV. Second, the presence of zero-return that are multiplied twice (with the previous and the next intraday return) leads to a downward bias of the BPV. Some alternative jump-robust measures have been proposed in the literature to deal with the aforementioned issues. Among them, the median realized RV (MedRV) (Andersen et al., (2012)) is very promising, as it has better properties in realistic settings and remains intuitive and easy to implement. ${ }^{4}$ The MedRV estimator reads as follows:

$$
M e d R V_{d, M}=\frac{\pi}{6-4 \sqrt{3}+\pi}\left(\frac{M}{M-2}\right) \sum_{j=2}^{M-1} \operatorname{med}\left(\left|r_{d, j-1}\right|,\left|r_{d, j}\right|,\left|r_{d, j+1}\right|\right)^{2}
$$

With the MedRV estimator, the impact of jumps completely vanishes except in the case of two consecutive jumps (which is extremely rare at the sampling frequencies used in empirical applications). In addition, the estimator is more robust to the occurrence of zero-returns except when many zero-returns are likely to be consecutive. We thus decide to consider the MedRV as a competitive alternative for our analysis and will provide all our results for both the BPV and the MedRV estimators.

For these three estimators of realized volatility, theory suggests that returns should be computed at the highest possible frequency, so that estimators converge asymptotically towards the true conditional volatility. However, it is well-known since Andersen and Bollerslev (1997, 1998) and Taylor and $\mathrm{Xu}$ (1997) that microstructure noise (due to price discreteness, bid-ask spread, non-synchronous trading, etc. $)^{5}$ may impact the realized volatility estimator at high frequency. To deal with this issue while making our results comparable with the rest of the literature, we follow the 5 minutes 'rule-of-thumb'. As our four series are highly liquid assets, this sampling interval is adequate to make our realized measures not to be impacted by the noise.

\footnotetext{
${ }^{3}$ This notation is used consistently in the paper.

${ }^{4}$ We could use other estimators to obtain measures of integrated variance, such as QRV (Christensen et al., 2009) estimator, which are shown to be more robust in the presence of microstructure noise and zero-returns. A comparison of these estimators and their properties for density forecasting is beyond the scope of this paper and left for future research.

${ }^{5}$ See Hansen and Lunde (2006) for a thorough discussion of this issue and Andersen et al. (2011) for a theoretical and empirical analysis of the impact of microstructure noise on the forecast of realized volatility. To deal with this issue, we use staggered versions of BPV and MedRV as advocated in Huang and Tauchen (2005).
} 
In what follows, we only present results for the MedRV estimator of realized volatility for the sake of space. Results using the BPV estimator are qualitatively similar and available from the authors upon request.

\subsection{Summary statistics}

Table 1 displays descriptive statistics regarding our returns and volatility measures. From the analysis of the table, returns on the four available assets are showing features that are consistent with the stylized facts usually reported in the empirical financial literature: the distribution of their returns are asymmetric and leptokurtic. They show different levels of volatilities: the WTI's volatility is the largest, reaching 37\%, when the 10-year bond futures' is $7.2 \%$. All skewness are negative over the portion of history investigated here. Kurtosis are all higher than 3, while still reaching various levels. The distribution with the fatter tails is the bond futures one, while the one with the thinner tails is the USDCAD one. The table also provides information regarding the distribution of the two volatility measures that we will use in our empirical experiments: the RV and MedRV measures are variance estimates, and the table displays statistics for their square root, so that to focus on volatility-like measures. Several comments can be raised from this second part of the table. First, the average level of our volatility measures is lower than the standard deviation of returns, especially in the case of the MedRV measure: for the S\&P500 index, the returns' standard deviation is $20.1 \%$ when the average square root of the RV is $13.1 \%$ and $12.4 \%$ in the case of MedRV. Similar cases can be raised from the other three assets. Second, the volatility skewness is positive, as it is distributed on the positive part of the real line. Third, the kurtosis is larger than 3 , and its largest reading is obtained in the case of the S\&P 500. These features are consistent for both the RV and the MedRV results.

\begin{tabular}{llcccc}
\hline & & S\&P 500 & 10-year futures & USDCAD & WTI \\
\hline \multirow{4}{*}{ Returns statistics } & Average return & 0.087 & 0.013 & 0.004 & 0.072 \\
& Volatility of returns & 0.201 & 0.072 & 0.079 & 0.376 \\
& Skewness & -1.984 & -3.376 & -0.150 & -1.493 \\
& Kurtosis & 72.849 & 87.579 & 10.057 & 31.039 \\
\hline \multirow{5}{*}{ RV statistics } & Average volatility - RV & 0.131 & 0.045 & 0.048 & 0.236 \\
& Volatility of volatility - RV & 0.095 & 0.021 & 0.027 & 0.119 \\
& Skewness of volatility - RV & 13.355 & 2.964 & 2.208 & 2.668 \\
& Kurtosis of volatility - RV & 458.792 & 36.097 & 11.747 & 22.075 \\
\hline \multirow{5}{*}{ MedRV statistics } & Average volatility - MedRV & 0.124 & 0.043 & 0.045 & 0.221 \\
& Volatility of volatility - MedRV & 0.091 & 0.019 & 0.026 & 0.114 \\
& Skewness volatility - MedRV & 11.195 & 1.626 & 2.423 & 2.915 \\
& Kurtosis of volatility - MedRV & 330.545 & 8.080 & 14.124 & 29.043 \\
\hline
\end{tabular}

Table 1: Descriptive statistics

\subsection{Detecting jumps}

Our methodology closely follows Boudt et al. (2011) which highlights the importance of consider intraweek periodicity for jump detection. Their methodology has been used in Lahaye et al. (2011). The estimation of intraweek periodicity is inspired by the early estimator of Taylor and Xu (1997). Boudt et al. (2011) modifies the estimator to make more robust to 
the presence of jumps in the intraday data. The estimated intraweek periodicity is then used to test for jumps following the methodology developed in Lee and Mykland (2008) (see also Andersen et al. (2007b)). In particular, we consider the test statistic:

$$
J_{t, i} \equiv \frac{\left|r_{t, i}\right|}{\widehat{\sigma_{t, i}}}
$$

where $r_{t, i}$ is the $i^{t h}$ return in day $t$ and $\sigma_{t, i}$ is the latent volatility at that time. $\sigma_{t, i}$ is estimated using an estimator which is robust to jumps.

While Boudt et al. (2011) follow Lee and Mykland (2008) in choosing the BPV esimator, we opt for the MedRV which has better finite-sample properties developed in Andersen et al. (2012). The window on which the robust-to-jumps estimator has to be computed is a tricky question (see the discussion in Lahaye et al. (2011) Section 2.1). We follow Andersen et al. (2007b) and use the estimated volatility over the day $t$. Lee and Mykland (2008) suggests to use the distribution of the statistic's maximum to conclude on the presence of jumps. In particular, if

$$
J_{t, i}>G_{-1}(1-\alpha) S_{n}+C_{n}
$$

with $n$ the total number of intraday returns in the full sample (number of days times number of intraday returns each day) and

$$
\begin{aligned}
& G_{-1}(1-\alpha) \text { the } 1-\alpha \text { the quantile function of the standard Gumbel distribution } \\
& \qquad C_{n}=\sqrt{2 \log n}-\frac{\log \pi+\log (\log n)}{2 \sqrt{2 \log n}} \text { and } S_{n}=\frac{1}{\sqrt{2 \log n}}
\end{aligned}
$$

The $1-\alpha$ quantile function of the standard Gumbel distribution is given by $-\log (-\log (1-\alpha))$.

We now introduce periodicity considerations in the original estimator of Lee and Mykland (2008) following Boudt et al. (2011). The main idea is to consider that the conditional volatility $\sigma_{t, i}$ is the product of a slowly-varying component $\delta_{t, i}$ and a deterministic circadian component $f_{t, i}$ whose aim is to model the intraweek periodicity. As in Taylor and Xu (1997), Boudt et al. (2011) assume that this deterministic component of the variance process sums to one:

$$
\int_{t-1}^{t} f^{2}(s) d s=1
$$

The modified version of the Lee and Mykland's test according to Boudt et al.' work is given as:

$$
\text { Filt } J_{t, i} \equiv \frac{\left|r_{t, i}\right|}{\widehat{\delta_{t, i}} \widehat{f_{t, i}}}
$$

where $\widehat{\delta_{t, i}}$ corresponds to the daily MedRV and $\widehat{f_{t, i}}$ is the estimated circadian component. 
The computation of the circadian component is as follows. First, we standardize intraday returns to allow for comparability across sample days. Intraday returns are standardized using MedRV for reasons presented above (Boudt et al. (2011) rely on the BPV estimator). Let the standardized $i^{t h}$ intraday return for day $t$ be

$$
\bar{r}_{t, i}=\frac{r_{t, i}}{\sqrt{M e d R V_{t}^{\Delta}}}
$$

Second, we use the nonparametric Weighted Standard Deviation (WSD) which has better efficiency under normality than classical estimators. The WSD nonparametric periodicity estimator for the $i^{\text {th }}$ intraday return in the sample days is given by:

$$
\hat{f}_{i}^{\mathrm{WSD}}=\frac{\mathrm{WSD}_{i}}{\sqrt{\frac{1}{M} \sum_{j=1}^{M} \mathrm{WSD}_{j}^{2}}}
$$

with

$$
\mathrm{WSD}_{j}=\sqrt{1.081 \times \frac{\sum_{h=1}^{N_{j}} w_{h, j} \bar{r}_{h, j}^{2}}{\sum_{h=1}^{N_{j}} w_{h, j}}}
$$

where the weights are given by $w_{h, j}=w\left(\bar{r}_{h, j} / \hat{f}_{j}^{\text {ShortH }}\right)$ with a weighting function $w(z)=1$ if $z^{2} \leq 6.635$ and 0 otherwise. 6.635 represents the $99 \%$ quantile of the $\chi^{2}(1)$ distribution.

$$
\hat{f}_{i}^{\text {ShortH }}=\frac{\text { ShortH }_{i}}{\sqrt{\frac{1}{M} \sum_{j=1}^{M} \text { ShortH }_{j}^{2}}}
$$

The standardization in Eq. (13) ensures that the standardization condition in Eq. (10) is met.

The Shortest Half (ShortH) is defined as:

$$
\operatorname{ShortH}_{j}=0.741 \times \min \left(\bar{r}_{\left(h_{j}\right), j}-\bar{r}_{(1), j}, \bar{r}_{\left(h_{j}+1\right), j}-\bar{r}_{(2), j}, \ldots, \bar{r}_{\left(n_{j}\right), j}-\bar{r}_{n_{j}-h_{j}+1, j}\right)
$$

where $\bar{r}_{(1), j}, \ldots, \bar{r}_{\left(n_{j}\right), j}$ are the ordered standardized intraday returns for the $j^{\text {th }}$ period of each day such that $\bar{r}_{(1), j}<\bar{r}_{(2), j}<\ldots<\bar{r}_{\left(n_{j}\right), j}$.

We implement this methodology and allow for a different periodicity each day of the week and each year over the full sample. From Figure 1, we observe a weekly profile that is very similar to the one in Boudt et al. (2011) and which exemplifies the variations in trading activity over the all week. Jumps detected for the four series are plotted in Figures A.1 to A.4. From the all Figures, it is clear that jumps tend to cluster in more volatile periods. Table 2 provides descriptive statistics for jumps detected using the Boudt et al.'s (2011) methodology. The number of days where a jump occurs is in line with the estimates in Andersen et al. (2007a) as it is in a range of 4 to $8 \%$. 

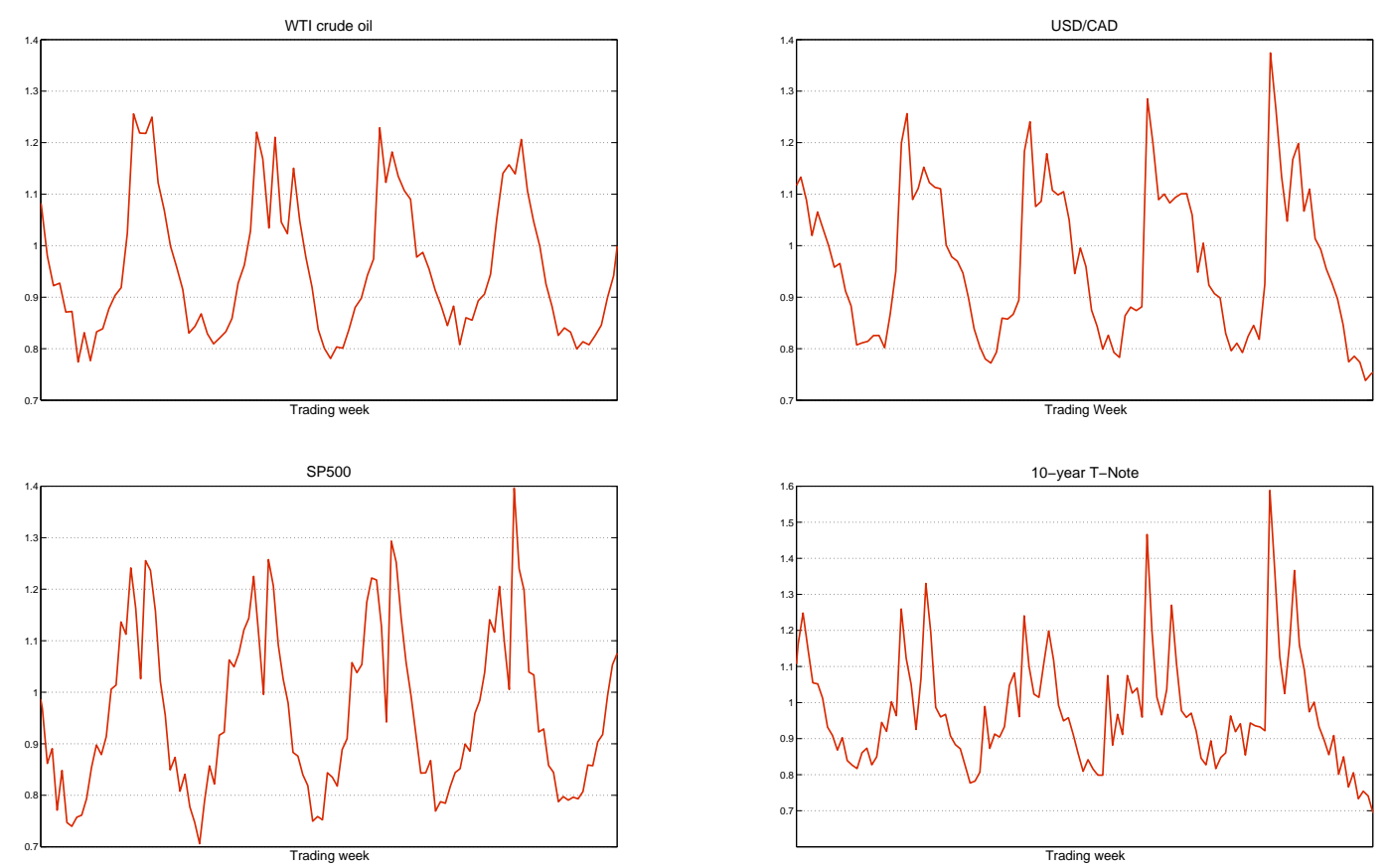

Figure 1: WSD periodicity for the four assets.

\begin{tabular}{cccccc}
\hline & Ann. Average & Ann. Std. Dev. & Skewness & Kurtosis & \% of jumpy days \\
\hline S\&P 500 & -0.296 & 0.125 & 0.308 & 5.825 & 0.03942 \\
WTI & -0.562 & 0.228 & 0.190 & 2.833 & 0.03934 \\
USDCAD & 0.040 & 0.044 & 0.263 & 4.131 & 0.0757 \\
TY & -0.036 & 0.057 & 1.310 & 13.433 & 0.0543 \\
\hline
\end{tabular}

Table 2: Descriptive statistics of the jump dataset

\section{Fitting the distribution of intraday jumps}

In standard asset pricing models jumps are usually characterized by a compound Poisson process such as in Merton (1976)'s model: the number of jumps follows a Poisson distribution and the jump size is distributed after a continuous distribution such as the Gaussian or the double exponential distributions (see Kou and Wang (2004)). The type of jumps that are obtained with the intraday methodologies described earlier present features that turns their empirical distribution incompatible with that of continuous distributions. With those methodologies, jumps are infrequent and potentially large. Therefore, a distribution with thin tails should not be able to pass a goodness-of-fit test comparing it to a sample of jumps. Second, jumps are estimated by thresholding intraday returns: this means that it is very unlikely that the absolute value of the estimated jumps will reach a very low value. The empirical distribution of jumps will therefore take the shape of a curve with two peaks: one for the jumps with a negative value and one for the jumps with positive ones. With these facts in mind, standard 
distributions - even the fat tailed ones - will not be able to pass an adequation test to such data-sets and another modeling direction must be explored. Descriptive statistics for our jumps data-sets are presented in Table 2: jumps are exhibiting asymmetry and leptokurticity levels that are not compatible with a Gaussian distribution. Then, Figure 2 displays the empirical distribution of jumps estimated from a non parametric estimator: the two peaks shape is obvious from those figures. In this section, we present our empirical approach to model the distribution of the jump sizes.

SP500

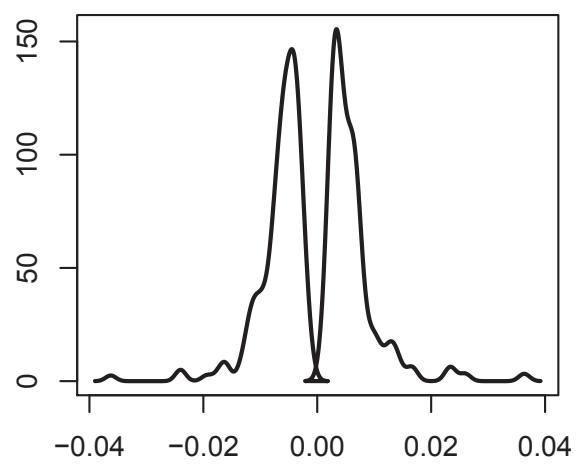

WTI

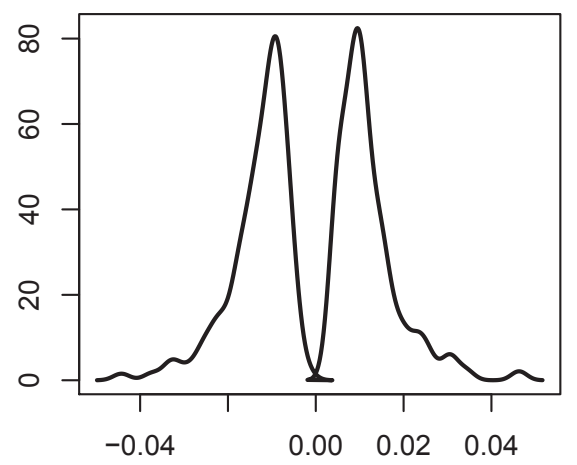

USDCAD

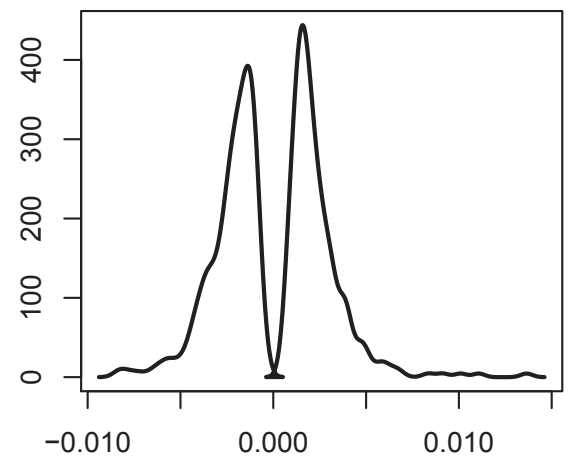

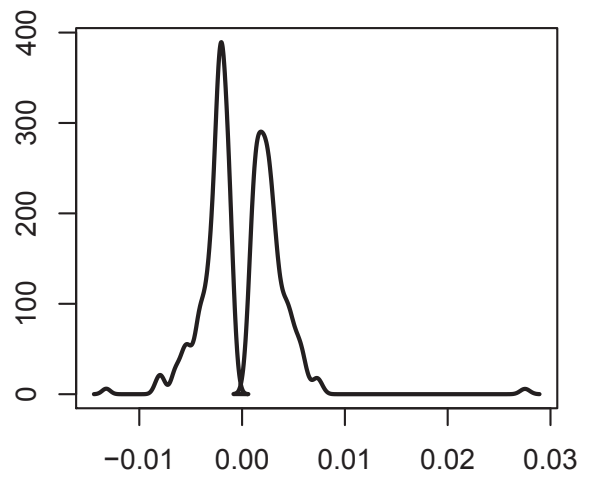

Figure 2: Empirical distribution of jumps for S\&P 500, WTI, T-notes and USDCAD exchange rate. The empirical distribution has been estimated using a non-parametric kernel. 


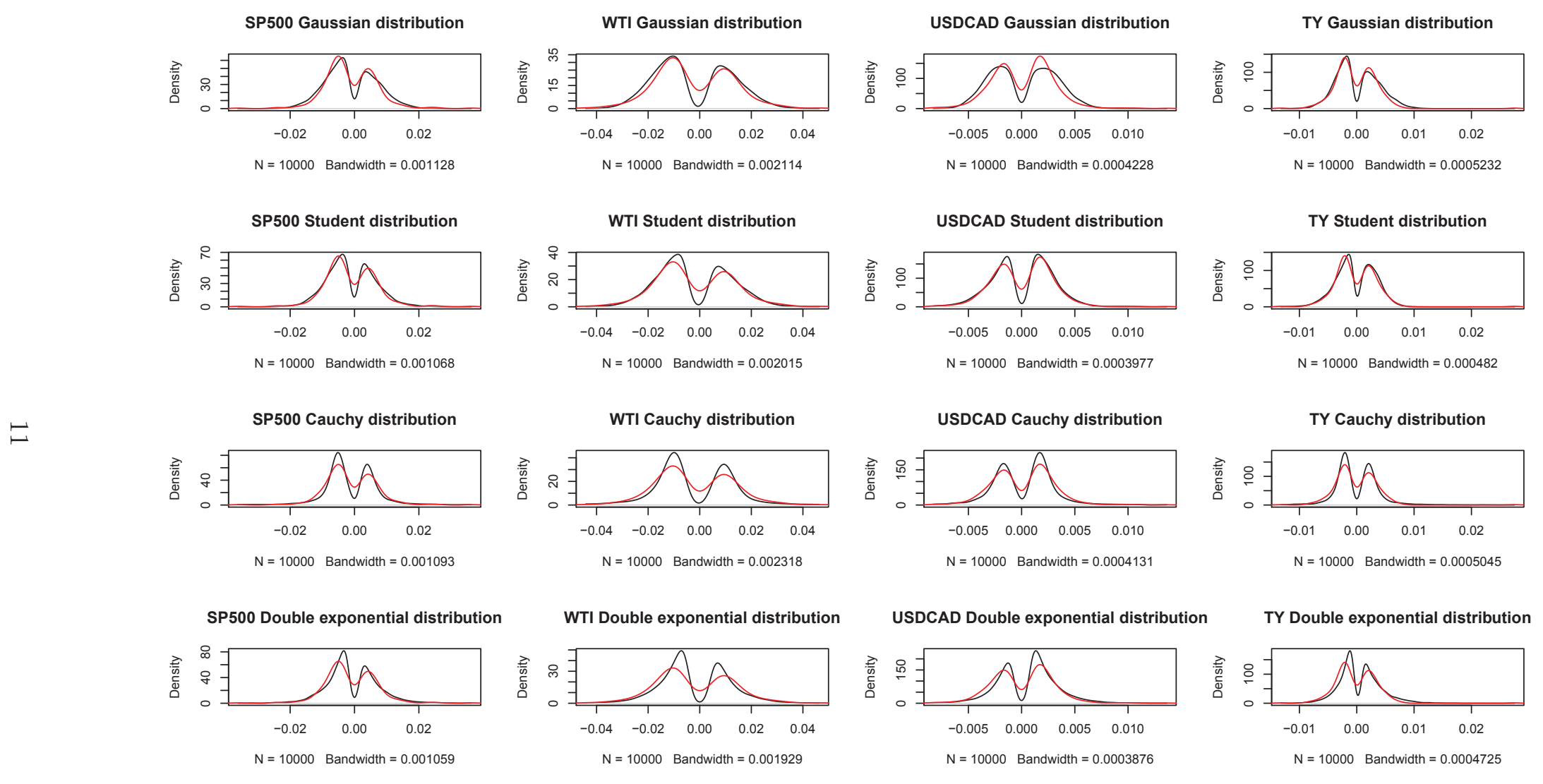

Figure 3: Empirical distribution of jumps for S\&P 500, WTI, T-notes and USDCAD exchange rate. The empirical distribution has been estimated using a non-parametric kernel. 
For the sake of clarity in this section, we focus only on dates for which a jump has been detected and we denote by $\mathcal{N}$ such a set of dates. In the following, we suppose that the jumps $\left(J_{t}\right)_{t \in \mathcal{N}}$ are i.i.d random variables and we denote by $f_{J}: \mathbb{R} \rightarrow \mathbb{R}_{+}$the corresponding density function. From Figure 2 we clearly see that $f_{J}$ is not only bimodal but also very small in a vicinity $] a: b[$ of zero. In order to reproduce this empirical feature we propose the following natural candidate:

$$
f_{J}(x)=\lambda \frac{f_{1}\left(x, \theta_{1}\right) 1_{x<a}}{\int_{-\infty}^{a} f_{1}\left(x, \theta_{1}\right) d x}+(1-\lambda) \frac{f_{2}\left(x, \theta_{2}\right) 1_{x>b}}{\int_{b}^{+\infty} f_{2}\left(x, \theta_{2}\right) d x}
$$

where $\lambda \in] 0,1\left[\right.$ and where $f_{1}$ and $f_{2}$ are two arbitrary density functions depending on two vectorial parameters $\theta_{1}$ and $\theta_{2}$. If we denote by $F_{1}$ (resp. $F_{2}$ ) the distribution function associated to $f_{1}$ (resp. $f_{2}$ ) we can write

$$
f_{J}(x)=\lambda \frac{f_{1}\left(x, \theta_{1}\right) 1_{x<a}}{F_{1}(a)}+(1-\lambda) \frac{f_{2}\left(x, \theta_{2}\right) 1_{x>b}}{1-F_{2}(b)}
$$

and $f_{J}$ may be simply seen as a mixture of left-right truncated distributions. In order to offer the maximum flexibility in terms of goodness-of-fit given the jumps' asymmetric and leptokurtic distribution as well as their two-peak properties, the set of parameters $\theta_{1}$ and $\theta_{2}$ do not need to be the same (even, $f_{1}$ and $f_{2}$ do not need to belong to the same family of distributions). In addition with the mixture and the truncation parameters, $f_{J}$ also depends on $\theta_{1}$ and $\theta_{2}$ and we favor four different potential candidates for $f_{i}, i \in 1,2$ selected for their parameter parsimony as well as their ability to fit the jumps' tail behaviors: the Gaussian distribution (mainly as a benchmark for leptokurticity), the scaled Student distribution ${ }^{6}$., the Cauchy distribution ${ }^{7}$ and the double exponential distributions ${ }^{8}$. Some of them have been used for modeling jump sizes - especially the first and the last one - when the other two have the ability to better fit tail behaviors. We discarded candidates coming from the Generalized hyperbolic distribution family: with 5 parameters, this distribution would require 12 parameters to be estimated when adding the threshold parameters $a$ and $b$. We want to rely on models that stand a chance to pass goodness-of-fit tests while remaining as parsimonious as possible.

\footnotetext{
${ }^{6}$ In this case $\theta_{i}=\left(\nu_{i}, \sigma_{i}\right) \in \mathbb{R}_{+}^{*} \times \mathbb{R}, f_{i}\left(x, \theta_{i}\right)=\frac{1}{\sigma_{i} \sqrt{\nu_{i}} \mathrm{~B}\left(\frac{1}{2}, \frac{\nu_{i}}{2}\right)}\left(1+\frac{x^{2}}{\sigma_{i}^{2} \nu_{i}}\right)^{-\frac{\nu_{i}+1}{2}}$ where B is the Beta function (beta using R) and $\forall x \in \mathbb{R}_{+}$(the negative values are obtained by symmetry), $F_{i}(x)=1-\frac{\tilde{\mathrm{B}}\left(\frac{\sigma_{i}^{2} \nu_{i}}{x^{2}+\sigma_{i}^{2} \nu_{i}}, \frac{\nu_{i}}{2}, \frac{1}{2}\right)}{2 \mathrm{~B}\left(\frac{\nu_{i}}{2}, \frac{1}{2}\right)}$ where $\tilde{\mathrm{B}}$ is the incomplete Beta function (Ibeta using the zipfR package)

${ }^{7}$ In this case, $\theta_{i}=\left(a_{i}, x_{i}^{0}\right) \in \mathbb{R}_{+}^{*} \times \mathbb{R}, f\left(x, \theta_{i}\right)=\frac{1}{\pi}\left[\frac{a_{i}}{\left(x-x_{i}^{0}\right)^{2}+a_{i}^{2}}\right]$ and $F_{i}(x)=\frac{1}{\pi} \arctan \left(\frac{x-x_{i}^{0}}{a_{i}}\right)+\frac{1}{2}$.

${ }^{8}$ In this case $\theta_{i}=\left(\mu_{i}, b_{i}\right) \in \mathbb{R} \times \mathbb{R}_{+}^{*}, f\left(x, \theta_{i}\right)=\frac{1}{2 b_{i}} \exp \left(-\frac{\left|x-\mu_{i}\right|}{b_{i}}\right)$ and $F_{i}(x)=$ $\begin{cases}\frac{1}{2} \exp \left(\frac{x-\mu_{i}}{b_{i}}\right) & \text { if } x<\mu_{i} \\ 1-\frac{1}{2} \exp \left(-\frac{x-\mu_{i}}{b_{i}}\right) & \text { if } x \geq \mu_{i}\end{cases}$
} 


\begin{tabular}{cccccc}
\hline & Single Gaussian & Gaussian & Scaled Student & Cauchy & Double exponential \\
\hline S\&P 500 & $0 \%$ & $9 \%$ & $41 \%$ & $96 \%$ & $4 \%$ \\
WTI & $0 \%$ & $16 \%$ & $80 \%$ & $84 \%$ & $1 \%$ \\
USDCAD & $0 \%$ & $0 \%$ & $31 \%$ & $67 \%$ & $0 \%$ \\
TY & $0 \%$ & $2 \%$ & $14 \%$ & $82 \%$ & $0 \%$ \\
\hline
\end{tabular}

Table 3: Test results for the Kolmogorov-Smirnov goodness-of-fit test. The table reports the p-values associated to the null hypothesis of an adequation of the proposed distribution to the time series.

The parameters of each distribution is estimated over each jump dataset full sample by numerically maximizing the log-likelihood associated to each model. We then run a KolmogorovSmirnov test for each of the models and obtain the results presented in Table 3. "Single Gaussian" refers to a simple Gaussian distribution fitted to each time series. The single Gaussian distribution is rejected for the four datasets, as it is neither asymmetric nor bimodal. The Gaussian bi-model distribution is rejected as well: it fails at capturing the tail behavior of the jumps' distribution. The Double exponential distribution is always rejected at a $5 \%$ risk level. Finally, the Cauchy and the scaled Student distributions are always accepted at a $5 \%$ risk level. However the acceptance probability are consistently higher for the Cauchy case: for this reason ${ }^{9}$, we will rely on this distribution throughout the forthcoming empirical analysis. Figure 3 displays the empirical distribution of the estimated models vs. the empirical distribution of the estimated jumps, for a more graphical assessment of the Kolmogorv-Smirnov test's results.

\section{The models}

\subsection{Linking conditional variance and realized volatility}

An important idea in Maheu and McCurdy (2011) is to relate the conditional variance of daily returns $\sigma_{t}^{2}$ to the realized volatility estimator through a cross-equation restriction. BarndorffNielsen and Shephard (2002) and Andersen et al. (2003) show that under some empirically realistic assumptions, the conditional variance of daily returns should be equal to the conditional expectation of quadratic variation with respect to the past information. ${ }^{10}$ Assuming that the realized volatility $R V_{t}$ is an unbiased estimator of the quadratic variation, it follows that:

$$
\sigma_{t}^{2}=E_{t-1}\left(R V_{t}\right)
$$

In other words, the one-period-ahead conditional expectation of the realized volatility should equal the "true" conditional volatility assuming the unbiasedness of the realized volatility estimator. Under the assumption of a log-normal distribution for the realized volatility ${ }^{11}$, the

\footnotetext{
${ }^{9}$ The fact that the scaled Student distribution both rely on parameters distributed over the real line and over $\mathbb{N}$ could also be problematic for the rolling estimation procedure that we intend on using.

${ }^{10}$ These ideas were already developed in papers such as French et al. (1987) or Zhou (1996).

${ }^{11}$ Empirical evidence of this hypothesis can be found in early contribution such as Andersen et al. (2001a and b, 2003). Similar evidence for foreign exchange rates, futures markets, crude oil futures and the FTSE index may be found in Pong et al. (2004), Thomakos and Wang (2003), Wang et al. (2008) and Areal and
} 
conditional expectation may be simply written as:

$$
\sigma_{t}^{2}=E_{t-1}\left(R V_{t}\right)=\exp \left(E_{t-1} \log \left(R V_{t}\right)+\frac{1}{2} \operatorname{Var}_{t-1}\left(\log \left(R V_{t}\right)\right)\right) .
$$

Using the results presented in Section 2, we can extend this theory by writing the realized volatility RV as the sum of the median realized volatility MedRV and the jump component quadratic variation when the latter is significant:

$$
\sigma_{t}^{2}=E_{t-1}\left(M e d R V_{t}+\sum_{j=J(t-1)}^{J(t)} \kappa^{2}\left(t_{j}\right)\right)
$$

where

$$
E_{t-1}\left(M e d R V_{t}\right)=\exp \left(E_{t-1} \log \left(M e d R V_{t}\right)+\frac{1}{2} \operatorname{Var}_{t-1}\left(\log \left(M e d R V_{t}\right)\right)\right) .
$$

Hence, if we are interested in the impact of disentangling jumps from the rest of the volatility, using Eq. 20 constitutes an appropriate way to proceed.

We now turn to the specification of a predictive model for realized volatility measures, namely the HAR model. Note that the choice of this model is central for the role that conditional expectation in Eq. (19) and (20) will play in forecasting.

\subsection{Heterogeneous autoregressive model of realized volatility}

The HAR-RV model initially developed by Corsi (2009) has been used with success in a number of recent contributions (Andersen et al. (2007a), Corsi et al. (2008), Liu and Maheu (2009), Duong and Swanson (2015), Patton and Sheppard (2015) among many others). The economic intuition behind this model is that different groups of investors have different investment horizons, and consequently behave differently (see Müller et al. (1997) for the presentation of the HARCH original model relying on the Heterogeneous Hypothesis). The genuine HAR-RV model is formally a constrained AR(22) model using RV as the realized measures of variance but the HAR can naturally accommodate all realized measures (as MedRV) and transformations of these measures. ${ }^{12}$ In particular, the log transformation has been found to perform very well and we will it in conjunction with our different realized measures $R M$. The HAR model using daily, weekly and monthly ${ }^{13}$ realized volatility components may be written as follows:

$$
\log \left(R M_{t}\right)=\omega+\phi_{1} \log \left(R M_{t-1}\right)+\phi_{2} \log \left(R M_{t-5: t-1}\right)+\phi_{3} \log \left(R M_{t-22: t-1}\right)+u_{t}
$$

where $\log \left(R M_{t-k: t-1}\right)=\frac{1}{k} \sum_{j=1}^{k} \log \left(R M_{t-j}\right)$.

\footnotetext{
Taylor (2002), respectively.

${ }^{12}$ Forsberg and Ghysels (2007) and Ghysels and Sohn (2009), note that other power transformations may be used to model the dynamics of the realized volatility. These studies show that for a number of stochastic volatility processes used in the financial literature the absolute value of the realized volatility is a better predictor of the future realized volatility, particularly for longer horizons. We do not follow this approach here.

${ }^{13}$ The optimal lag structure for the HAR model has been investigated in Craioveanu and Hillebrand (2010) who find that the genuine structure suggested in Corsi (2009) performs the best.
} 
In the following, the error term $u_{t}$ is supposed to be Gaussian ${ }^{14}$ to cope with 19 and 21.

\subsection{The bivariate model for daily returns and volatilities}

In this section we present the bivariate model inspired by Maheu and McCurdy (2011). The main interest behind multivariate models consists in obtaining densities forecasts. More precisely our approach is made of three key ingredients: an independent jump component ${ }^{15}$, a conditional variance (with or without jumps) that is linked to appropriate realized volatility measures and a conditionally Gaussian heterogeneous autoregressive model of realized volatility. Thus, in the model with at most one jump at time $t$ the dynamics of the log-returns may be written as

$$
r_{t}=\mu+\sigma_{t} \epsilon_{t}+\mathbb{1}_{t} J_{t}
$$

where $\sigma_{t}$ is made observable through the relation $\sigma_{t}^{2}=E_{t-1}\left(R M_{t}\right)$ with a well $\operatorname{chosen}^{16}$ realized measure RM fulfilling

$$
\log \left(R M_{t}\right)=\omega+\phi_{1} \log \left(R M_{t-1}\right)+\phi_{2} \log \left(R M_{t-5: t-1}\right)+\phi_{3} \log \left(R M_{t-22: t-1}\right)+\gamma_{1} \epsilon_{t-1}+\gamma_{2} \mathbb{1}_{t-1}\left|J_{t-1}\right|+u_{t} .
$$

In the preceding equation, $\gamma_{1}$ is a parameter capturing a feedback effect from past returns to the subsequent increment in volatility. Finally, $\gamma_{2}$ is meant to materialize the feedback effect from past jumps to increases in volatility ${ }^{17}$. In 23 and 24 we suppose that $\left(\left(\epsilon_{t}, \mathbb{1}_{t}, J_{t}, u_{t}\right)\right)_{t \in\{0, \ldots, T\}}$ are independent random variables and we denote by $\left(\mathcal{F}_{t}\right)_{t \in\{0, \ldots, T\}}$ the associated filtration ${ }^{18}$. Assuming that the $u_{t}$ are i.i.d $N(0,1)$ we have

$$
\sigma_{t}^{2}=E_{t-1}\left(R M_{t}\right)=\exp \left(E_{t-1} \log \left(R M_{t}\right)+\frac{1}{2} \operatorname{Var}_{t-1}\left(\log \left(R M_{t}\right)\right)\right) .
$$

Concerning the separated jump component we suppose that $\mathbb{1}_{t-1}$ follows a binomial distribution, with a time independent parameter $p$ representing the probability that a jump occurs at time $t$, and that the jumps $J_{t}$ follows the mixture of left-right truncated Cauchy distributions described in Section 3.

When $p=0$ (and thus $\mathrm{RM}=\mathrm{RV}$ ), this model nests the so-called HAR-RV model developed in Maheu and McCurdy (2011) and for our empirical investigations $\epsilon_{t}$ can either be Gaussian or a mixture of Gaussian distributions ${ }^{19}$, the later being able to span a very large scope of

\footnotetext{
${ }^{14}$ As in Maheu and McCurdy (2011), conditional non-normality is introduced in the bivariate modeling via return equation and not in the variance one.

${ }^{15}$ According to the empirical descriptive statistics about jumps extracted using the detection methodology developed in Section 2, we favor in this section a model with at most one jump where the jump distribution is carefully estimated using the parametric family of distributions described in Section 3.

${ }^{16}$ As explained in Section 4.1, when the jump probability $p$ is null we take $R M=R V$ and $R M=M e d R V$ otherwise.

${ }^{17}$ On the impact of jumps in returns on volatility see for example Duffie et al. (2000), Eraker et al. (2003), Eraker (2004), Maheu et McCurdy (2004), Aït-Sahalia et al. (2015) and Carr and Wu (2010) among others.

${ }^{18} \mathrm{We}$ can see easily that this filtration is also generated by the observations of the log-returns and of the realized measure RM until time $t$.

${ }^{19}$ The mixture of two Gaussian distributions has the density
}

$$
f_{M N}(x)=\alpha n\left(x, \mu_{1}, \sigma_{1}\right)+(1-\alpha) n\left(x, \mu_{2}, \sigma_{2}\right),
$$


couples of kurtosis and skewness as presented in Bertholon et al. (2006). In this case, using the independence hypothesis, we obtain the conditional joint density of the pair $\left(r_{t}, R V_{t}\right)$ and the bivariate model is estimated by conditional maximum-likelihood.

When $p \neq 0$ (and thus $\mathrm{RM}=$ MedRV), even if the conditional joint density of the pair $\left(r_{t}, R V_{t}\right)$ is still available, we favor a two-steps estimation strategy that takes advantage of the jump detection presented in Section 2. In fact, the probability $p$ is first estimated by the proportion of jumpy days in our datasets and the parameters of the jump density $f_{J}$ are obtained from the classical maximum-likelihood for independent observations. In a second step, remarking that the conditional density of the log-returns is given by

$$
f_{r_{t}}\left(z \mid \mathcal{F}_{t-1}\right)=p \int_{-\infty}^{+\infty} f_{\epsilon}\left(\frac{x-\mu}{\sigma_{t}}\right) f_{J}(z-x) \frac{d x}{\sigma_{t}}+(1-p) f_{\epsilon}\left(\frac{z-\mu}{\sigma_{t}}\right)
$$

where $f_{\epsilon}$ is the density of the innovations in the return equation, we obtain the remaining parameters using the explicit conditional joint density of the pair $\left(r_{t}, \operatorname{MedR} V_{t}\right)$ and conditional maximum-likelihood.

Across our empirical experiences, we focus on the following models:

- A HAR-RV-G model for which $\epsilon_{t} \sim N(0,1), p=0$ and $\gamma_{2}=0$

- A HAR-RV-MN model for which $\epsilon_{t} \sim M N\left(\alpha, \mu_{1}, \sigma_{1}, \mu_{2}, \sigma_{2}\right), p=0$ and $\gamma_{2}=0$

- A HAR-MedRV-MN model for which $\epsilon_{t} \sim M N\left(\alpha, \mu_{1}, \sigma_{1}, \mu_{2}, \sigma_{2}\right), p=0$ and $\gamma_{2}=0$

- A HAR-MedRV-Jump-No Feedback model for which $\epsilon_{t} \sim N(0,1), p \neq 0$ and $\gamma_{2}=0$

- A HAR-MedRV-Jump-Feedback model for which $\epsilon_{t} \sim N(0,1), p \neq 0$ and $\gamma_{2} \neq 0$.

\subsection{Test methodology}

To evaluate the relative accuracy of competing forecasts, we rely on the test statistics developed by Diebold and Mariano (1995) in the context of the comparison of density forecasts (Amisano and Giacomini (2007)). In our presentation we follow Maheu and McCurdy (2011), focusing on the ability of the approach to test multi-period forecasts.

For $M \in\{A, B\}$ we consider two competing models and we denote by $\theta^{M}$ the corresponding parameters that drive the log-returns and the intraday volatility measure dynamics. Starting from a sample $\left(r_{1}, \ldots r_{T}\right)$ of size $T$ we want to test forecast horizons $1 \leq k \leq k_{\max }$ through rolling-window forecasting schemes of size $\tau^{20}$. Thus, for $k \geq 1$, the average predictive likelihood is given by

where $\left(\alpha, \mu_{1}, \mu_{2}, \sigma_{1}, \sigma_{2}\right) \in[0,1] \times \mathbb{R}^{2} \times\left(\mathbb{R}^{*}\right)^{2}$ and where $n\left(., \mu_{i}, \sigma_{i}\right)$ is the density of a Gaussian random variable with expectation $\mu_{i}$ and standard deviation $\sigma_{i}$ (see for example Kon (1984), Akgiray and Booth (1987), Tucker and Pond (1998) and Alexander and Lazar (2006))

${ }^{20}$ In the empirical part, we estimate the models on a rolling window of 1,260 daily observations for all but the MCD series (700 observations). 


$$
D_{M, k}=\frac{1}{T-\tau-k_{\max }+1} \sum_{t=\tau+k_{\max }-k}^{T-k} \log f_{M, k}\left(r_{t+k}, \widehat{\theta_{t+k}^{M}} \mid \mathcal{F}_{t}\right),
$$

where $\widehat{\theta_{t+k}^{M}}$ is estimated using the sample $\left(r_{t-\tau-k_{\max }+1}, \ldots, r_{t}\right)$ and where

$$
f_{M, k}\left(r_{t+k}, \widehat{\theta_{t+k}^{M}} \mid \mathcal{F}_{t}\right)
$$

is the conditional density of the log-returns at time $t+k$, given $\mathcal{F}_{t}$ and $\widehat{\theta_{t+k}^{M}}$, evaluated at the realized log-return $r_{t+k}$. The particular form of (27) allow us to obtain a term structure of average predictive likelihoods, $\left(D_{M, 1}, \ldots, D_{M, k_{\max }}\right)$ to compare the performance of alternative models, $\mathrm{M}$, over an identical set of out-of-sample data points $\left(r_{\tau+k_{\max }}, \ldots, r_{T}\right)$.

In our setting the conditional densities $f_{M, k}\left(x, \widehat{\theta_{t+k}^{M}} \mid \mathcal{F}_{t}\right)$ do not have a closed form except for $k=1$ and have, in general, to be evaluated generating independent realizations of $r_{t+k}$ given $\mathcal{F}_{t}$ and using classical density kernel estimators. Nevertheless, remarking that

$$
f_{M, k}\left(r_{t+k}, \widehat{\theta_{t+k}^{M}} \mid \mathcal{F}_{t}\right)=\int_{\mathbb{R}_{+}} f_{M, k}\left(r_{t+k}, \widehat{\theta_{t+k}^{M}} \mid \sigma_{t+k}^{2}\right) p\left(\sigma_{t+k} \mid \mathcal{F}_{t}\right) d \sigma_{t+k}^{2}
$$

we have

$$
f_{M, k}\left(r_{t+k}, \widehat{\theta_{t+k}^{M}} \mid \mathcal{F}_{t}\right) \approx \frac{1}{N} \sum_{i=1}^{N} f_{M, k}\left(r_{t+k}, \widehat{\theta_{t+k}^{M}} \mid\left(\sigma_{t+k}^{2}\right)^{i}\right)
$$

where $f_{M, k}\left(y_{t+k}, \widehat{\theta_{t+k}^{M}} \mid \sigma_{t+k}^{2}\right)$ is now perfectly known and where $\left(\sigma_{t+k}^{2}\right)^{i}$ are independent realizations of $\sigma_{t+k}^{2}$ generated from the preceding dynamics with parameters $\widehat{\theta_{t+k}^{M}}$ and starting values $\sigma_{t}$ and $r_{t}$ as explained in Maheu and McCurdy (2011).

According to Amisano Giacomini (2007), under the null hypothesis of equal performance, the statistic based on predictive likelihoods of horizon $k$ for models $A$ and $B$,

$$
t_{A, B}^{k}=\frac{\left(D_{A, k}-D_{B, k}\right) \sqrt{T-\tau-k_{\max }+1}}{\hat{\sigma}_{A, B, k}}
$$

is asymptotically standard Normal, where $\hat{\sigma}_{A, B, k}$ is a properly selected estimator for the variance of

$$
\log f_{A, k}\left(r_{t+k}, \widehat{\theta_{t+k}^{A}} \mid \mathcal{F}_{t}\right)-\log f_{B, k}\left(r_{t+k}, \widehat{\theta_{t+k}^{B}} \mid \mathcal{F}_{t}\right) .
$$

Here, as proposed Amisano Giacomini (2007), we use a Newey-West estimator that take into account heteroskedasticity and autocorrelation. One of the main interest of this approach comes from the fact that the two models can be nested or not ${ }^{21}$ and can be estimated using very different techniques from the moment that they are based on a finite estimation window.

\footnotetext{
${ }^{21}$ In our empirical part some nested models as HAR-MedRV-Jump-Feedback and HAR-MedRV-Jump-No Feedback will be compared.
} 


\section{Empirical results}

Tables 4, 5, 6 and 7 present the estimation results for the four datasets used in this article: the S\&P 500, the WTI, the USDCAD and the the 10-year bond futures. The estimation has been performed over rolling windows of 1260 trading days, that is 5 years of data ${ }^{22}$. The tables display the average values for each parameters across our rolling estimations, as well as the 5 and $95 \%$ quantiles. For most of the parameters, both quantiles across our estimates are of the same sign, pointing into the direction of statistically significant parameters.

The $\gamma_{1}$ parameter is found to be negative and different from zero for the S\&P 500 and the WTI, highlighting that negative returns contribute positively to the subsequent volatility. In the case of bond futures and in the case of USDCAD, this parameter is not found to be statistically significant when the conditional distribution of returns is chosen to be asymmetric. This conclusion both holds for the mixture of Gaussian distribution and the discontinuous jump distribution introduced in Section 3. Overall, across all models, when using both types of non-Gaussian conditional distribution, the $\gamma_{1}$ parameter is found to be weaker. Now, when comparing the samples, leverage effects - that is an asymmetric feedback from past returns into subsequent volatilities - seem to be weaker for the US 10-year bond futures and the Canadian Dollar than for the rest of the assets considered here.

Turning to the jump-to-volatility feedback parameter $\gamma_{2}$, our results suggest that only in the case of the WTI is this parameter significantly different from zero. In the S\&P 500 case, the estimates vary from -4.807 to 58.218 : despite the negativity of the $5 \%$ quantile, this jump-tovolatility feedback might be playing a role as well. Turning finally to the jump parameters, our estimates depict distributions that are consistent with the descriptive statistics provided in Table 2: the jump distributions exhibit a negative average for all datasets but one, as the $\lambda$ parameter that is found to be greater than 0.5 across all samples. Its lowest value is obtained for the USDCAD dataset for which $\lambda$ is estimated to be equal to 0.52 while the average value of the jumps is 0.4. Then, the strongest asymmetry is found for the 10-year futures in Table 2 , and in Table 7 as it is the only dataset for which $\left|\mu_{a}\right|<\left|\mu_{b}\right|$. Also, the jump volatility estimates presented in Table 2 are well represented across the estimation tables: $\sigma_{a}, \sigma_{b}, a$ and $b$ have a higher absolute value in the case of the WTI and of the S\&P500, where jump volatility is the greatest. Finally, the frequency of the estimated jumps, i.e. the $p$ parameter of the Bernouilli distribution, is found to range between $7.8 \%$ in the USDCAD case to $3.8 \%$ in the WTI one: the estimated jumps are rare events indeed.

\footnotetext{
${ }^{22}$ Here, we use the same window length as in Maheu and MacCurdy (2011) given that our empirical methodology has been inspired by theirs.
} 


\begin{tabular}{|c|c|c|c|c|c|c|c|c|c|c|c|c|c|c|}
\hline \multicolumn{15}{|c|}{ RV - Gaussian } \\
\hline & $\omega$ & $\phi_{1}$ & $\phi_{2}$ & $\phi_{3}$ & $\gamma_{1}$ & & & & & & & & & \\
\hline Average value & -1.501 & 0.204 & 0.377 & 0.268 & -0.11 & & & & & & & & & \\
\hline $95 \%$ quantile & -0.446 & 0.344 & 0.551 & 0.479 & -0.072 & & & & & & & & & \\
\hline $5 \%$ quantile & -2.985 & 0.099 & 0.152 & 0.152 & -0.145 & & & & & & & & & \\
\hline \multicolumn{15}{|c|}{$\mathrm{RV}-\mathrm{MN}$} \\
\hline & $\omega$ & $\phi_{1}$ & $\phi_{2}$ & $\phi_{3}$ & $\gamma_{1}$ & $\alpha$ & $\mu_{1}$ & $\sigma_{1}$ & $\mu_{2}$ & $\sigma_{2}$ & & & & \\
\hline Average value & -1.58 & 0.21 & 0.377 & 0.262 & -0.103 & 0.325 & -0.308 & 0.888 & 0.37 & 1.318 & & & & \\
\hline $95 \%$ quantile & -0.532 & 0.334 & 0.549 & 0.448 & -0.069 & 0.50 & 0.277 & 1.19 & 0.677 & 1.636 & & & & \\
\hline $5 \%$ quantile & -2.985 & 0.113 & 0.188 & 0.163 & -0.131 & 0.023 & -1.058 & 0.472 & 0.07 & 1.125 & & & & \\
\hline \multicolumn{15}{|c|}{ MedRV - MN } \\
\hline & $\omega$ & $\phi_{1}$ & $\phi_{2}$ & $\phi_{3}$ & $\gamma_{1}$ & $\alpha$ & $\mu_{1}$ & $\sigma_{1}$ & $\mu_{2}$ & $\sigma_{2}$ & & & & \\
\hline Average value & -1.623 & 0.221 & 0.356 & 0.271 & -0.096 & 0.3 & 0.147 & 1.105 & 0.276 & 1.399 & & & & \\
\hline $95 \%$ quantile & -0.59 & 0.323 & 0.495 & 0.431 & -0.056 & 0.5 & 1.641 & 2.138 & 0.641 & 1.68 & & & & \\
\hline $5 \%$ quantile & -2.985 & 0.138 & 0.155 & 0.166 & -0.134 & 0.026 & -0.557 & 0.568 & -0.051 & 1.123 & & & & \\
\hline \multicolumn{15}{|c|}{ MedRV - Jumps - No feedback } \\
\hline & $\omega$ & $\phi_{1}$ & $\phi_{2}$ & $\phi_{3}$ & $\gamma_{1}$ & $\mathrm{a}$ & $\mathrm{b}$ & $\mu_{a}$ & $\mu_{b}$ & $\sigma_{a}$ & $\sigma_{b}$ & $\lambda$ & $p$ & \\
\hline Average value & -1.433 & 0.217 & 0.365 & 0.275 & -0.1 & -0.001 & 0.002 & -0.005 & 0.005 & 0.002 & 0.003 & 0.575 & 0.039 & \\
\hline $95 \%$ quantile & -0.469 & 0.371 & 0.504 & 0.436 & -0.053 & -0.001 & 0.003 & -0.004 & 0.007 & 0.003 & 0.005 & 0.632 & 0.055 & \\
\hline $5 \%$ quantile & -2.709 & 0.109 & 0.147 & 0.162 & -0.136 & -0.002 & 0.001 & -0.007 & 0.003 & 0.001 & 0.001 & 0.519 & 0.024 & \\
\hline \multicolumn{15}{|c|}{ MedRV - Jumps - Feedback } \\
\hline & $\omega$ & $\phi_{1}$ & $\phi_{2}$ & $\phi_{3}$ & $\gamma_{1}$ & $\gamma_{2}$ & $\mathrm{a}$ & $\mathrm{b}$ & $\mu_{a}$ & $\mu_{b}$ & $\sigma_{a}$ & $\sigma_{b}$ & $\lambda$ & $p$ \\
\hline Average value & -1.458 & 0.216 & 0.365 & 0.274 & -0.099 & 16.898 & -0.001 & 0.002 & -0.005 & 0.005 & 0.002 & 0.003 & 0.575 & 0.039 \\
\hline $95 \%$ quantile & -0.469 & 0.37 & 0.505 & 0.438 & -0.05 & 58.218 & -0.001 & 0.003 & -0.004 & 0.007 & 0.003 & 0.005 & 0.632 & 0.055 \\
\hline $5 \%$ quantile & -2.758 & 0.108 & 0.149 & 0.158 & -0.136 & -4.807 & -0.002 & 0.001 & -0.007 & 0.003 & 0.001 & 0.001 & 0.519 & 0.024 \\
\hline
\end{tabular}

Table 4: Parameters estimates for the HAR based models using the returns on the S\&P 500

The estimates presented in this table are obtained by maximizing the log-likelihood associated with of the models presented in Section 4 and using the S\&P 500 data. The estimation has been performed over a 1260 days rolling period. "Average" presents the average value obtained across the estimation. The table also displays the associated 5 and $95 \%$ quantiles. 
RV - Gaussian

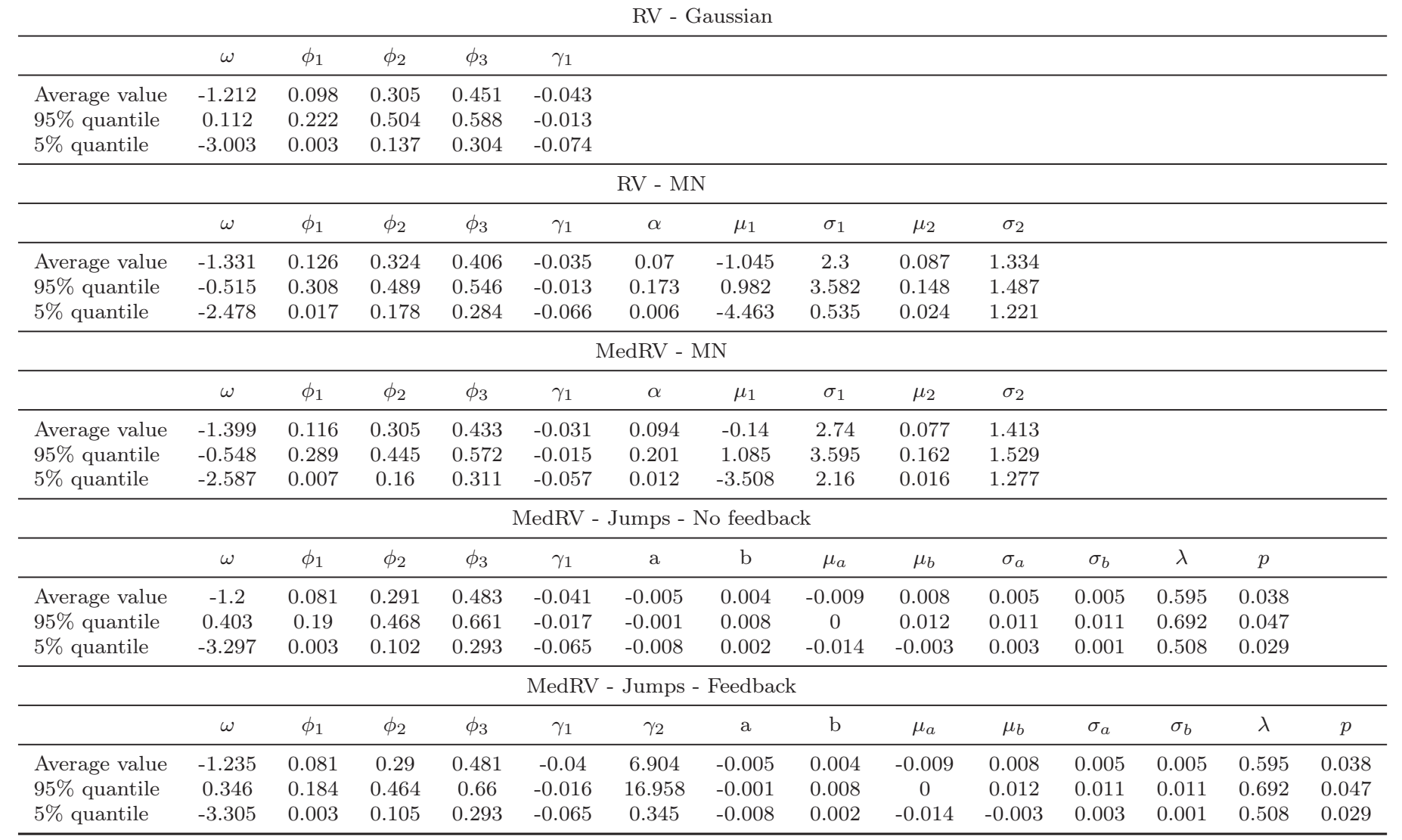

Table 5: Parameters estimates for the HAR based models using the returns on the WTI

The estimates presented in this table are obtained by maximizing the log-likelihood associated with of the models presented in Section 4 and using the WTI data. The estimation has been performed over a 1260 days rolling period. "Average" presents the average value obtained across the estimation. The table also displays the associated 5 and $95 \%$ quantiles. 
RV - Gaussian

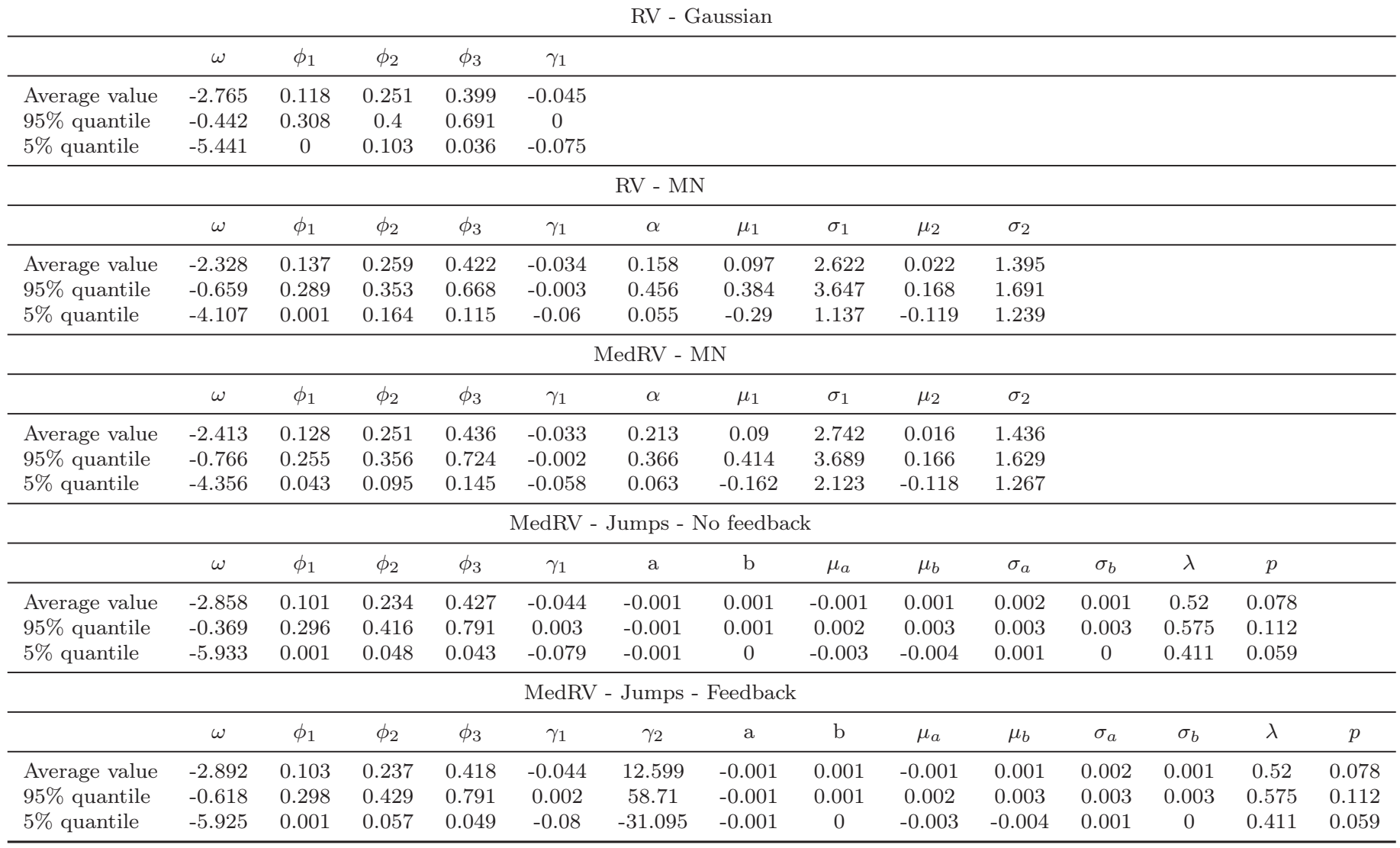

Table 6: Parameters estimates for the HAR based models using the returns on the USDCAD

The estimates presented in this table are obtained by maximizing the log-likelihood associated with of the models presented in Section 4 and using the USDCAD data The estimation has been performed over a 1260 days rolling period. "Average" presents the average value obtained across the estimation. The table also displays the associated 5 and $95 \%$ quantiles. 
RV - Gaussian

\begin{tabular}{|c|c|c|c|c|c|c|c|c|c|c|c|c|c|c|}
\hline \multicolumn{15}{|c|}{ RV - Gaussian } \\
\hline & $\omega$ & $\phi_{1}$ & $\phi_{2}$ & $\phi_{3}$ & $\gamma_{1}$ & & & & & & & & & \\
\hline Average value & -2.446 & 0.073 & 0.148 & 0.567 & -0.008 & & & & & & & & & \\
\hline $95 \%$ quantile & -0.827 & 0.229 & 0.308 & 0.8 & 0.009 & & & & & & & & & \\
\hline $5 \%$ quantile & -4.878 & 0 & 0 & 0.328 & -0.031 & & & & & & & & & \\
\hline \multicolumn{15}{|c|}{$\mathrm{RV}-\mathrm{MN}$} \\
\hline & $\omega$ & $\phi_{1}$ & $\phi_{2}$ & $\phi_{3}$ & $\gamma_{1}$ & $\alpha$ & $\mu_{1}$ & $\sigma_{1}$ & $\mu_{2}$ & $\sigma_{2}$ & & & & \\
\hline Average value & -2.369 & 0.113 & 0.181 & 0.515 & -0.002 & 0.028 & -3.654 & 7.397 & 0.092 & 1.337 & & & & \\
\hline $95 \%$ quantile & -0.718 & 0.243 & 0.301 & 0.787 & 0.013 & 0.081 & -0.148 & 31.008 & 0.186 & 1.457 & & & & \\
\hline $5 \%$ quantile & -4.528 & 0.048 & 0 & 0.327 & -0.019 & $0-15.703$ & 0.99 & 0.048 & 1.24 & & & & & \\
\hline \multicolumn{15}{|c|}{ MedRV - MN } \\
\hline & $\omega$ & $\phi_{1}$ & $\phi_{2}$ & $\phi_{3}$ & $\gamma_{1}$ & $\alpha$ & $\mu_{1}$ & $\sigma_{1}$ & $\mu_{2}$ & $\sigma_{2}$ & & & & \\
\hline Average value & -2.326 & 0.123 & 0.23 & 0.463 & -0.003 & 0.057 & -3.61 & 8.578 & 0.088 & 1.403 & & & & \\
\hline $95 \%$ quantile & -0.635 & 0.202 & 0.316 & 0.728 & 0.011 & 0.125 & 0.321 & 26.833 & 0.184 & 1.566 & & & & \\
\hline $5 \%$ quantile & -4.309 & 0.072 & 0.141 & 0.306 & -0.019 & 0.002 & -14.217 & 0.788 & 0.025 & 1.267 & & & & \\
\hline \multicolumn{15}{|c|}{ MedRV - Jumps - No feedback } \\
\hline & $\omega$ & $\phi_{1}$ & $\phi_{2}$ & $\phi_{3}$ & $\gamma_{1}$ & $\mathrm{a}$ & $\mathrm{b}$ & $\mu_{a}$ & $\mu_{b}$ & $\sigma_{a}$ & $\sigma_{b}$ & $\lambda$ & $p$ & \\
\hline Average value & -2.162 & 0.146 & 0.131 & 0.534 & -0.01 & -0.001 & 0.001 & -0.001 & 0.002 & 0.002 & 0.002 & 0.563 & 0.049 & \\
\hline $95 \%$ quantile & -0.58 & 0.384 & 0.277 & 0.833 & 0.01 & 0 & 0.001 & 0.001 & 0.002 & 0.003 & 0.002 & 0.623 & 0.066 & \\
\hline $5 \%$ quantile & -4.8 & 0.035 & 0 & 0.338 & -0.03 & -0.001 & 0.001 & -0.002 & 0 & 0.001 & 0.001 & 0.448 & 0.04 & \\
\hline \multicolumn{15}{|c|}{ MedRV - Jumps - Feedback } \\
\hline & $\omega$ & $\phi_{1}$ & $\phi_{2}$ & $\phi_{3}$ & $\gamma_{1}$ & $\gamma_{2}$ & $\mathrm{a}$ & $\mathrm{b}$ & $\mu_{a}$ & $\mu_{b}$ & $\sigma_{a}$ & $\sigma_{b}$ & $\lambda$ & $p$ \\
\hline Average value & -2.312 & 0.142 & 0.131 & 0.526 & -0.011 & 4.071 & -0.001 & 0.001 & -0.001 & 0.002 & 0.002 & 0.002 & 0.563 & 0.049 \\
\hline $95 \%$ quantile & -0.639 & 0.377 & 0.277 & 0.834 & 0.009 & 37.485 & 0 & 0.001 & 0.001 & 0.002 & 0.003 & 0.002 & 0.623 & 0.066 \\
\hline $5 \%$ quantile & -5.1 & 0.032 & 0 & 0.334 & -0.031 & -22.575 & -0.001 & 0.001 & -0.002 & 0 & 0.001 & 0.001 & 0.448 & 0.04 \\
\hline
\end{tabular}

Table 7: Parameters estimates for the HAR based models using the returns on the US 10-year futures

The estimates presented in this table are obtained by maximizing the log-likelihood associated with of the models presented in Section 4 and using the US 10-year bond futures. The estimation has been performed over a 1260 days rolling period. "Average" presents the average value obtained across the estimation. The table also displays the associated 5 and $95 \%$ quantiles. 
SP500

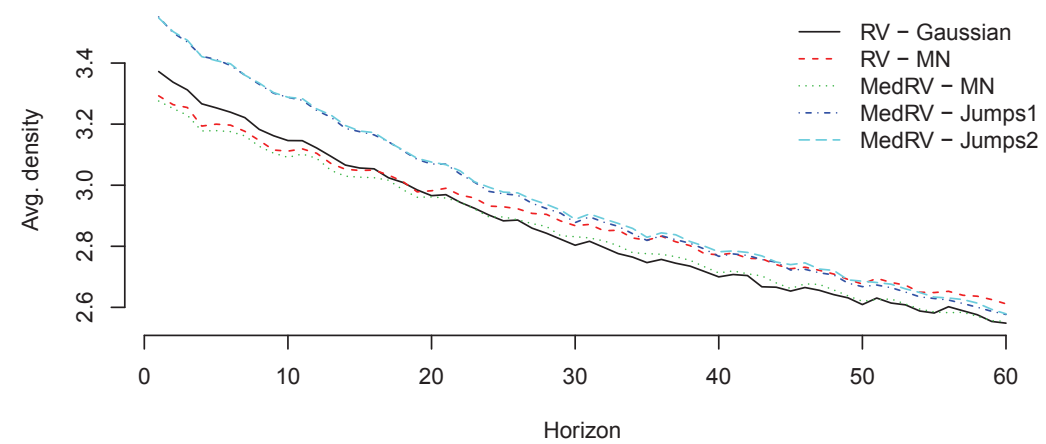

10-year rates futures

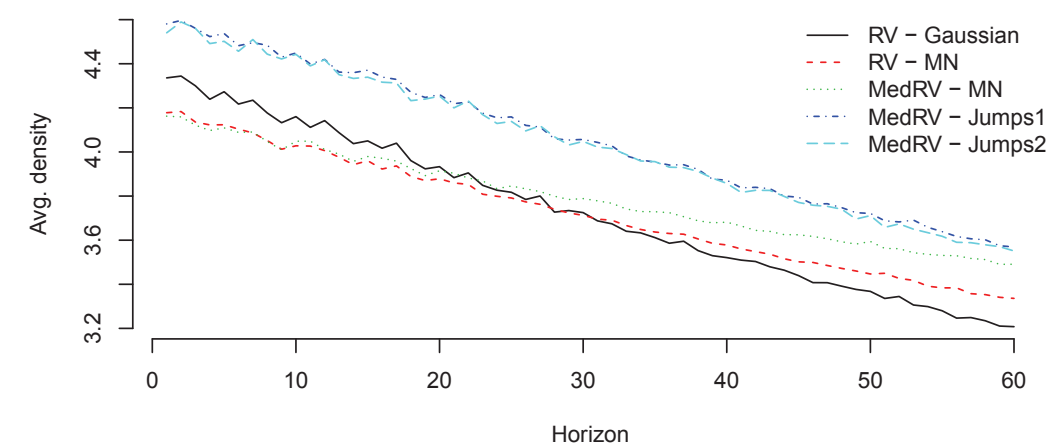

USDCAD

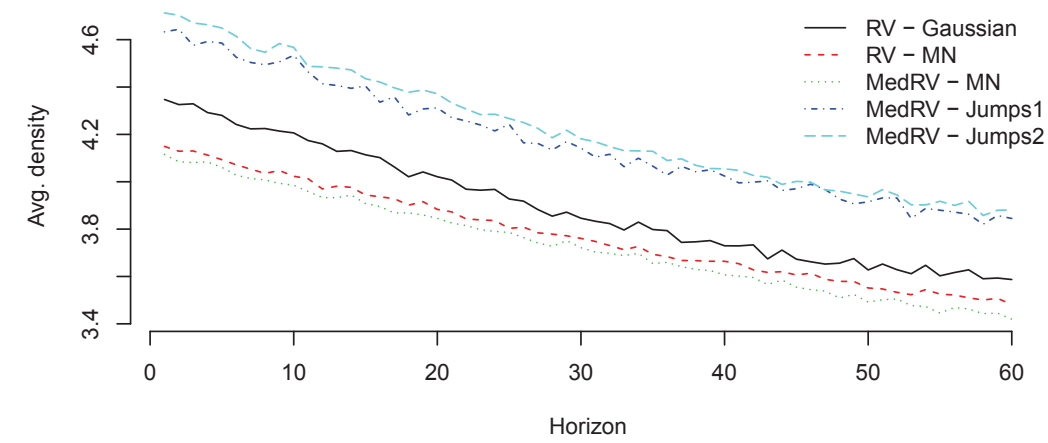

WTI

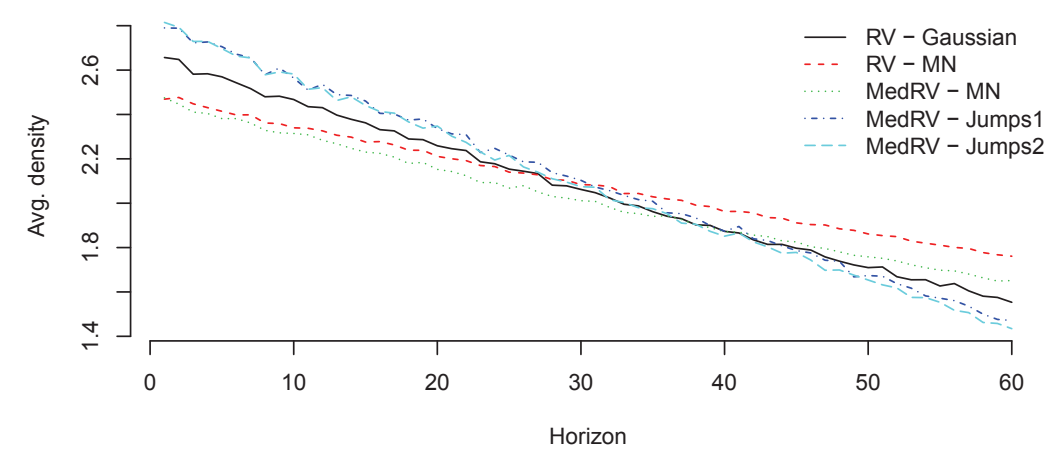

Figure 4: Average log density per forecasting horizon for each model across the four datasets. 
Tables 8, 9, 10 and 11 and Figure 4 display the results of the Amisano and Giacomini (2007) forecasting density test. From these tables and graphs, several conclusions can be raised. First, for forecast horizons ranging from 1 and 30 days, the models using the MedRV volatility measure as well as jumps based on the discontinuous distributions introduced earlier statistically dominate the rest of the models across the four datasets. This conclusion is the main conclusion of this article: for forecasting horizon of up to a month and a half, disentangling jumps from volatility improve our ability to forecast the distribution of the four very diverse types of returns on financial assets that are considered in this article. Second, the jump-to-volatility feedback component does not improve the forecast ability of the models for all assets but in the cases of a forecast horizon of 60 days for USDCAD and for the WTI, for which a statistically significant parameter had been found: the jump-to-volatility feedback component only seems to matter in the case of oil prices for forecast horizons of 20 and 60 days. In the case of oil, for distant horizon density forecasts, the Gaussian RV model still outperforms the rest of its competitors. Finally, in the case of the S\&P 500, for a 60-day forecast horizon the Gaussian RV model dominates the rest of tested models. Still, for such a forecast horizon, the jump-based models remain equivalent to the MedRV-MN model, a sign that the jump estimation methodologies yield accurate jump estimates.

\begin{tabular}{|c|c|c|c|c|c|}
\hline \multicolumn{6}{|c|}{ Horizon $=5$ days } \\
\hline & RV-G & RV-MN & MedRV-MN & MedRV-Jump 1 & MedRV-Jump 2 \\
\hline RV-G & & -2.3 & -2.37 & 46.14 & 49.29 \\
\hline RV-MN & & & -2.09 & 14.55 & 14.7 \\
\hline MedRV-MN & & & & 12.22 & 12.29 \\
\hline MedRV-Jump 1 & & & & & -0.32 \\
\hline MedRV-Jump 2 & & & & & \\
\hline \multicolumn{6}{|c|}{ Horizon $=20$ days } \\
\hline & $R V-G$ & RV-MN & MedRV-MN & MedRV-Jump 1 & MedRV-Jump 2 \\
\hline RV-G & & 0.22 & -1.4 & 25.56 & 24.64 \\
\hline RV-MN & & & -1.85 & 23.17 & 25.66 \\
\hline MedRV-MN & & & & 17.21 & 18.06 \\
\hline MedRV-Jump 1 & & & & & 0.98 \\
\hline MedRV-Jump 2 & & & & & \\
\hline \multicolumn{6}{|c|}{ Horizon $=60$ days } \\
\hline & $R V-G$ & RV-MN & MedRV-MN & MedRV-Jump 1 & MedRV-Jump 2 \\
\hline RV-G & & 13.74 & 8.38 & 4.43 & 3.31 \\
\hline RV-MN & & & -6.12 & -4.65 & -4.16 \\
\hline MedRV-MN & & & & -1.18 & -1.06 \\
\hline MedRV-Jump 1 & & & & & -0.25 \\
\hline MedRV-Jump 2 & & & & & \\
\hline
\end{tabular}

Table 8: S\&P 500 forecasting test results

This table presents the Amisano and Giacomini (2007) forecasting density test results for various forecasting horizons, ranging from 5 to 60 days. The table reads as follow: for a forecasting horizon of 5 days and when comparing the density forecasts obtained with a MedRV-Jump 2 model vs. a MedRV-MN model, the statistics is equal to 12.29. The MedRV-Jump 2 model is therefore found to dominate the MedRV-MN model given the sample used here. 


\begin{tabular}{|c|c|c|c|c|c|}
\hline \multicolumn{6}{|c|}{ Horizon $=5$ days } \\
\hline & $R V-G$ & RV-MN & MedRV-MN & MedRV-Jump 1 & MedRV-Jump 2 \\
\hline RV-G & & -5.99 & -5.68 & 46.52 & 48.72 \\
\hline RV-MN & & & -1.14 & 28.03 & 27.96 \\
\hline MedRV-MN & & & & 26.3 & 26.88 \\
\hline MedRV-Jump 1 & & & & & -1.68 \\
\hline MedRV-Jump 2 & & & & & \\
\hline \multicolumn{6}{|c|}{ Horizon $=20$ days } \\
\hline & $R V-G$ & $\mathrm{RV}-\mathrm{MN}$ & MedRV-MN & MedRV-Jump 1 & MedRV-Jump 2 \\
\hline RV-G & & -7.52 & -9.15 & 31.08 & 34.15 \\
\hline RV-MN & & & -4.35 & 32.22 & 44.97 \\
\hline MedRV-MN & & & & 33.57 & 48.53 \\
\hline MedRV-Jump 1 & & & & & -1.76 \\
\hline \multicolumn{6}{|l|}{ MedRV-Jump 2} \\
\hline \multicolumn{6}{|c|}{ Horizon $=60$ days } \\
\hline & RV-G & $\mathrm{RV}-\mathrm{MN}$ & MedRV-MN & MedRV-Jump 1 & MedRV-Jump 2 \\
\hline RV-G & & -3.57 & -5.5 & 13.18 & 9.8 \\
\hline RV-MN & & & -2.65 & 15.34 & 13.92 \\
\hline MedRV-MN & & & & 16.88 & 14.98 \\
\hline MedRV-Jump 1 & & & & & -2.61 \\
\hline MedRV-Jump 2 & & & & & \\
\hline
\end{tabular}

Table 9: USDCAD forecasting test results

This table presents the Amisano and Giacomini (2007) forecasting density test results for various forecasting horizons, ranging from 5 to 60 days. The table reads as follow: for a forecasting horizon of 5 days and when comparing the density forecasts obtained with a MedRV-Jump 2 model vs. a MedRV-MN model, the statistics is equal to 26.88. The MedRV-Jump 2 model is therefore found to dominate the MedRV-MN model given the sample used here. 


\begin{tabular}{|c|c|c|c|c|c|}
\hline \multicolumn{6}{|c|}{ Horizon $=5$ days } \\
\hline & RV-G & RV-MN & MedRV-MN & MedRV-Jump 1 & MedRV-Jump 2 \\
\hline RV-G & & -4.8 & -5.13 & 33.29 & 29.32 \\
\hline RV-MN & & & -7.7 & 11.54 & 9.92 \\
\hline MedRV-MN & & & & 10.39 & 9.11 \\
\hline MedRV-Jump 1 & & & & & -1.04 \\
\hline MedRV-Jump 2 & & & & & \\
\hline \multicolumn{6}{|c|}{ Horizon $=20$ days } \\
\hline & $\mathrm{RV}-\mathrm{G}$ & $\mathrm{RV}-\mathrm{MN}$ & MedRV-MN & MedRV-Jump 1 & MedRV-Jump 2 \\
\hline RV-G & & -1.64 & -5.25 & 10.49 & 7.9 \\
\hline RV-MN & & & -17.31 & 7 & 5.34 \\
\hline MedRV-MN & & & & 9.46 & 8.73 \\
\hline MedRV-Jump 1 & & & & & -2.87 \\
\hline \multicolumn{6}{|l|}{ MedRV-Jump 2} \\
\hline \multicolumn{6}{|c|}{ Horizon $=60$ days } \\
\hline & RV-G & RV-MN & MedRV-MN & MedRV-Jump 1 & MedRV-Jump 2 \\
\hline RV-G & & 15.11 & 12.6 & -7.33 & -9.14 \\
\hline RV-MN & & & -21.32 & -12.17 & -12.57 \\
\hline MedRV-MN & & & & -11.04 & -11.53 \\
\hline MedRV-Jump 1 & & & & & -2.91 \\
\hline MedRV-Jump 2 & & & & & \\
\hline
\end{tabular}

Table 10: WTI forecasting test results

This table presents the Amisano and Giacomini (2007) forecasting density test results for various forecasting horizons, ranging from 5 to 60 days. The table reads as follow: for a forecasting horizon of 5 days and when comparing the density forecasts obtained with a MedRV-Jump 2 model vs. a MedRV-MN model, the statistics is equal to 9.11. The MedRV-Jump 2 model is therefore found to dominate the MedRV-MN model given the sample used here. 


\begin{tabular}{|c|c|c|c|c|c|}
\hline \multicolumn{6}{|c|}{ Horizon $=5$ days } \\
\hline & $R V-G$ & RV-MN & MedRV-MN & MedRV-Jump 1 & MedRV-Jump 2 \\
\hline RV-G & & -5.55 & -4.1 & 25.28 & 24 \\
\hline RV-MN & & & 6.58 & 15.29 & 13.68 \\
\hline MedRV-MN & & & & 14.84 & 13.86 \\
\hline MedRV-Jump 1 & & & & & -1.4 \\
\hline MedRV-Jump 2 & & & & & \\
\hline \multicolumn{6}{|c|}{ Horizon $=20$ days } \\
\hline & $R V-G$ & $\mathrm{RV}-\mathrm{MN}$ & MedRV-MN & MedRV-Jump 1 & MedRV-Jump 2 \\
\hline RV-G & & -9.35 & -0.12 & 35.14 & 33.01 \\
\hline RV-MN & & & 11.21 & 31.95 & 27.12 \\
\hline MedRV-MN & & & & 35.02 & 28.4 \\
\hline MedRV-Jump 1 & & & & & 0.66 \\
\hline MedRV-Jump 2 & & & & & \\
\hline \multicolumn{6}{|c|}{ Horizon $=60$ days } \\
\hline & RV-G & $\mathrm{RV}-\mathrm{MN}$ & MedRV-MN & MedRV-Jump 1 & MedRV-Jump 2 \\
\hline RV-G & & 5.77 & 12.95 & 23.57 & 27.46 \\
\hline RV-MN & & & 8.33 & 17.03 & 20.98 \\
\hline MedRV-MN & & & & 16.34 & 16.28 \\
\hline MedRV-Jump 1 & & & & & 1.05 \\
\hline MedRV-Jump 2 & & & & & \\
\hline
\end{tabular}

Table 11: 10-year futures forecasting test results

This table presents the Amisano and Giacomini (2007) forecasting density test results for various forecasting horizons, ranging from 5 to 60 days. The table reads as follow: for a forecasting horizon of 5 days and when comparing the density forecasts obtained with a MedRV-Jump 2 model vs. a MedRV-MN model, the statistics is equal to 13.86. The MedRV-Jump 2 model is therefore found to dominate the MedRV-MN model given the sample used here. 


\section{Concluding remarks}

With the growing recognition that point forecasts, the traditional focus, are better seen as the central points of ranges of uncertainty, in recent years increased emphasis has been given to density forecasts. More generally, it is important to provide a quantitative indication of the uncertainty associated with a point forecast, along with the balance of risks (skewness) on the upside and downside and the probability of extreme events (fat tails or kurtosis). This is achieved by publishing a density forecast. Importantly, the density forecast gives any users of the forecast an indication, in advance, of the health risks associated with its use.

Based on a set of nested competing models, this article aims at testing whether disentangling jumps from volatility using intraday datasets helps forecast the density of returns. Using four different datasets - equities, bonds, commodities and currencies - we find consistent evidence that for short to medium term horizons, the density forecasts based on such an approach improves over its competitors. This result is obtained by relying on a jump modeling that takes into account the specificities of the intraday-based jump estimates. Whilst numerous authors have considered the informational content of continuous vs. jump components for volatility forecasting, none have thought to address the particular question of density forecasting. Detection of such information for different classes of assets would indicate the ability of new econometric models to anticipate the evolution of density returns in a fundamentally different way compared to more traditional forecasting models.

As potential extensions to the present work, other jump detection techniques may be used (see Christensen et al. (2010) among others). Nevertheless, collectively taken, our results are sufficiently strong so that we can believe they would be robust to alternative jump detection methods. Another potential extension would be to consider additional economic variables such as in Christiansen et al. (2012) or Paye (2012). 


\section{References}

Aït-Sahalia, Y., J. Cacho-Diaz, and Laeven, R. J., 2015. Modeling financial contagion using mutually exciting jump processes. Journal of Financial Economics 117, 585-606.

Akgiray, V. And G.G. Bоотh, 1987. Compound distribution models of stock returns: An empirical comparison. Journal of Financial Research 10, 269-280.

Alexander, C. And E. Lazar, 2006. Normal mixture GARCH(1,1): Applications to exchange rate modeling. Journal of Applied Econometrics 21, 307-336.

Amisano, G. and R. Giacomini, 2007. Comparing density forecasts via weighted likelihood ratio tests. Journal of Business \& Economic Statistics 25, 177-190.

Andersen, T. G., Bollerslev, T., 1997. Intraday periodicity and volatility persistence in financial markets. Journal of Empirical Finance 4, 115-158.

Andersen, T.G., Bollerslev, T., 1998. Answering the skeptics: yes, standard volatility models do provide accurate forecasts. International Economic Review 39, 885-905.

Andersen, T.G., T. Bollerslev, F.X. Diebold and H. Ebens, 2001a. The distribution of stock return volatility. Journal of Financial Economics 61, 43-76.

Andersen, T.G., T. Bollerslev, F.X. Diebold and P. Labys, 2001b. The distribution of exchange rate volatility. Journal of the American Statistical Association 96, 42-55.

Andersen, T.G., T. Bollerslev, F.X. Diebold and P. Labys, 2003. Modeling and forecasting realized volatility. Econometrica 71, 579-625.

Andersen, T.G., T. Bollerslev and F.X. Diebold, 2007a. Roughing it up: Including jump components in the measurement, modeling and forecasting of return volatility. Review of Economics and Statistics 89, 701-720.

Andersen, T.G., T. Bollerslev and D. Dobrev, 2007b. No-arbitrage semi-martingale restrictions for continuous-time volatility models subject to leverage effects, jumps and i.i.d. noise: Theory and testable distributional implications. Journal of Econometrics 138, 125-180.

Andersen, T.G., Bollerslev, T., Frederiksen, P., Nielsen, M.O., 2010. Continuous-time models, realized volatilities, and testable distributional implications for daily stock returns. Journal of Applied Econometrics 25, 233-261.

Andersen, T.G., Bollerslev, T., Meddahi, N., 2011. Realized volatility forecasting and market microstructure noise. Journal of Econometrics 160, 220-234.

Andersen, T.G., D. Dobrev and E. Schaumburg, 2012. Jump-robust volatility estimation using nearest neighbor truncation. Journal of Econometrics 169, 75-93.

Ané, T., Geman, H., 2000. Order flow, transaction clock, and normality of asset returns. Journal of Finance $55,2259-2284$.

Areal, N. And S.J. TAYlor, 2002. The realized volatility of FTSE-100 futures prices. Journal of Futures Markets 22, 627-648.

Barndorff-Nielsen, O. And N. Shephard, 2002. Econometric analysis of realized volatility and its use in estimating stochastic volatility models. Journal of the Royal Statistical Society, Series B 64, 253-280. 
Barndorff-Nielsen, O., Shephard, N., 2004. Power and bipower variation with stochastic volatility and jumps. Journal of Financial Econometrics 2, 1-37.

Barndorff-Nielsen, O., Shephard, N., 2006. Econometrics of testing for jumps in financial economics using bipower variation. Journal of Financial Econometrics 4, 1-30.

Bertholon, H., A. Monfort and F. Pegoraro, 2006. Pricing and inference with mixtures of conditionally normal processes. SSRN Working Paper, no. 997975.

Bollerslev, Litvinova, J., Tauchen, G., 2006. Leverage and volatility feedback effects in high-frequency data. Journal of Financial Econometrics 4, 353-384.

Bollerslev, T., Kretschmer, U., Pigorsch, C., Tauchen, G., 2009. A discrete-time model for daily S\&P500 returns and realized variations: jumps and leverage effects. Journal of Econometrics 150, 151-166.

Boudt, K., C. Croux and S. Laurent, S., 2011. Robust estimation of intraweek periodicity in volatility and jump detection. Journal of Empirical Finance 18, 353-367.

CARR, P. AND L. Wu, 2011. Leverage effect, volatility feedback, and self-exciting market disruptions. Bloomberg Portfolio Research Paper, (2009-03).

Christensen, K., Oomen, R., Podolskij, M., 2010. Realised quantile-based estimation of the integrated variance. Journal of Econometrics 159, 74-98.

Christiansen, C., M. Schmeling And A. Schrimpf, 2012. A comprehensive look at financial volatility prediction by economic variables. Journal of Applied Econometrics 27, 956-977.

Christoffersen, P., B. Feunou, K. Jacobs and N. Meddahi, 2014. The economic value of realized volatility: Using high-frequency returns for option valuation. Journal of Financial and Quantitative Analysis 49, 663-697.

Clements, M.P., 2005. Evaluating Econometric Forecasts of Economic and Financial Variables. Basingstoke and London: Palgrave Macmillan.

Clements, M.P., A.B. Galvao And J.H. Kim, 2008. Quantile forecasts of daily exchange rate returns from forecasts of realized volatility. Journal of Empirical Finance 15, 729-750.

Corradi, V. and N.R. Swanson, 2006. Predictive density evaluation. In: G. Elliot, C.W.J. Granger and A. Timmermann (Eds.), Handbook of Economic Forecasting, vol. 1, Elsevier.

Corsi, F., 2009. A simple approximate long memory model of realized volatility. Journal of Financial Econometrics 7, 174-196.

Corsi, F., S. Mittnik, C. Pigorsch And U. Pigorsch, 2008. The volatility of realized volatility. Econometric Reviews 27, 46-78.

Corsi, F., Pirino, D., Renò, R., 2010. Threshold bipower variation and the impact if jumps on volatility forecasting. Journal of Econometrics 159, 276-288.

Craioveanu, M. and E. Hillebrand, 2010. Why it is O.K. to use the HAR-RV $(1,5,21)$ model. Unpublished manuscript.

Diebold, F.X. and R.S. Mariano, 1995. Comparing predictive accuracy. Journal of Business \& Economic Statistics 13, 253-263.

Duffie, D., J. Pan and K. Singleton, 2000. Transform analysis and asset pricing for affine jump-diffusions. Econometrica 68, 1343-1376. 
Dumitru, A.-M., Urga, G., 2012. Identifying jumps in financial assets: A comparison between nonparametric jump tests. Journal of Business \& Economic Statistics 30, 242-255.

Duong, D. And N.R. Swanson, 2015, Empirical evidence on the importance of aggregation, asymmetry, and jumps for volatility prediction. Journal of Econometrics 187, 606-621.

Elliott, G. And A. Timmermann, 2016. Economic Forecasting. Princeton University Press, Princeton, NJ.

Eraker, B., M. Johannes and N. Polson, 2003. The impact of jumps in equity index volatility and returns. Journal of Finance 58, 1269-1300.

Eraker, B., 2004. Do stock prices and volatility jump? Reconciling evidence from spot and option prices. Journal of Finance 59, 1367-1403.

Fleming, J., C. Kirby and B. Ostdiek, 2003. The economic value of volatility timing using realized volatility. Journal of Financial Economics 67, 473-509.

Forsberg, L. And E. Ghysels, 2007. Why do absolute returns predict volatility so well? Journal of Financial Econometrics 5, 31-67.

French, K.R., G.W. Schwert and R.F. Stambaugh, 1987. Expected stock returns and volatility. Journal of Financial Economics 19,3-29.

Ghysels, E. And B. Sohn, 2009. Which power variation predicts volatility well? Journal of Empirical Finance $16,686-700$.

Hansen, P.R. And A. Lunde, 2006. Realized variance and market microstructure noise. Journal of Business and Economic Statistics 24, 127-218.

Hansen, P.R., Huang, Z., Shek, H.H., 2011. Realized GARCH: a joint model for returns and realized measures of volatility. Journal of Applied Econometrics 27, 877-906.

Huang, X. and G.Tauchen, 2005. The relative price contribution of jumps to total price variance. Journal of Financial Econometrics 3, 456-499.

Kon, S. J., 1984. Models of stock returns: A comparison. Journal of Finance 39, 147-165.

Kou, S.G. And H. WAnG, 2004. Option pricing under a double exponential jump diffusion model. Management Science 50, 1178-1192.

Lahaye, J., Laurent, S., Neely, C.J., 2010. Jumps, cojumps, and macro announcements. Journal of Applied Econometrics 26, 893-921.

Lee, S.S., Mykland, P.A., 2008. Jumps in financial markets: A new nonparametric test and jump dynamics. Review of Financial Studies 21, 2535-2563.

Liu, C. And J.M. Maheu, 2009. Forecasting realized volatility: A Bayesian model-averaging approach. Journal of Applied Econometrics 24, 709-733.

Maheu, J. M. And T.H. McCurdy, 2004. News arrival, jump dynamics, and volatility components for individual stock returns. Journal of Finance 59, 755-793.

Maheu, J. M. And T.H. McCurdy, 2011. Do high-frequency measures of volatility improve forecasts of return distributions? Journal of Econometrics 160, 69-76. 
Merton, R., 1976. Option pricing when underlying stock returns are discontinuous. Journal of Financial Economics 3, 125-144.

Müller, U.A., M.M.Dacorogna, R.D. Davé, R.B., Olsen and O.V. Pictet, 1997. Volatilities of different time resolutions - Analyzing the dynamics of market components. Journal of Empirical Finance 4, 213-239.

Oomen, R.C.A., 2006. Properties of realized variance under alternative sampling schemes. Journal of Business \& Economic Statistics 24, 219-237.

PAYE, B.S., 2012. Déjà vol: predictive regressions for aggregate stock market volatility using macroeconomic variables. Journal of Financial Economics 106, 527-546.

Patton, A.J., Sheppard, K., 2015. Good volatility, bad volatility: Signed jumps and the persistence of volatility. Review of Economics and Statistics 97, 683-697.

Pong, S., M.B. Shackleton, S.J. Taylor and X. Xu, 2004. Forecasting currency volatility: A comparison of implied volatilities and AR(FI)MA models. Journal of Banking and Finance 28, 2541-2563.

Shephard N. And K. Sheppard, 2010. Realising the future: Forecasting with high-frequency-based volatility (HEAVY) models. Journal of Applied Econometrics 25, 197-231.

TAY, A.S. And K.F. Wallis, 2000. Density forecasting: a survey. Journal of Forecasting 19, 235-54.

TAYlor, S.J. And X. Xu, 1997. The incremental volatility information in one million foreign exchange quotations. Journal of Empirical Finance 4, 317-340.

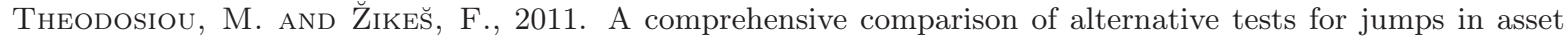
prices. Working Paper 2011-2, Central bank of Cyprus, July.

Thomakos, D.D. And T. WAng, 2003. Realized volatility in the futures markets. Journal of Empirical Finance 10, 321-353.

Timmermann, A., 2006. Forecast combinations. In Elliott, G., Granger, C.W.J. and Timmermann, A. (Eds.), Handbook of Economic Forecasting Volume 1, 135-96. Amsterdam: North-Holland.

Tucker, A.L. And L. Pond, 1998. The probability distribution of foreign exchange price changes: Tests of candidate processes. Review of Economics and Statistics 11, 638-647.

Wallis, K.F., 2007. Forecast uncertainty, its representation and evaluation. In: Mariano, R.S., Tse, Y.K. (Eds.), Econometric Forecasting and High-Frequency Data Analysis Volume 13, 1-51. Lecture Notes Series of the Institute for Mathematical Sciences, National University of Singapore. Singapore: World Scientific.

WAng, T., J. Wu And J. YAng, 2008. Realized volatility and correlation in energy futures markets. Journal of Futures Markets 28, 993-1011.

Zноu, B., 1996. High frequency data and volatility in foreign-exchange rates. Journal of Business \& Economic Statistics 14, 45-52. 


\section{Appendix A - Data}

The S\&P 500 futures data is a very liquid contract traded on the CME which is a tradable asset in opposition with the underlying S\&P 500 cash index. The time span for the S\&P 500 futures is from January 1, 1996 to July 31, 2008 (3,192 trading days originally). As is usual, we consider the continuous series of the front month contract using a rollover procedure which selects the largest volume each day to jump from one contract to the next. ${ }^{23}$. Trading of the S\&P 500 futures contract occurs from 8:30 AM to 3:15 PM which should, on normal days, provide 81 intraday returns when using a sampling interval of 5 minutes. We remove days with less than 81 returns which is generally an indication of part closure of the trading place. We check that all accepted days have a sufficient number of transactions, and a limited number of zero-returns as well. After cleaning the data, we obtain a sample of 3,135 days. For these selected days, the average number of trades for the continuous rollover series is 3,090 (the total number of ticks is equal to $9,809,697$ ). We observe a relative stability of the number of transactions each day during the whole period.

The 10-year U.S. Treasury Note futures is the second asset and received a treatment very similar to that of the S\&P500 futures.

The USD/CAD exchange rate is the third asset for our study. As in previous contributions (see Andersen et al. (2001) among many others), we consider the period going from 9:00 PM to 8:59 PM the next day as a unit period, because the FX market is opened on a 24-hour window.

Our fourth asset is the West Texas Intermediate (WTI) light sweet crude oil futures contract, traded on the New York Mercantile Exchange (NYMEX) now a branch of CME. The rollover series is built as explained previously for the S\&P 500. The period considered is from October 8, 2001 to January 15, 2010. The WTI contract is one of the most traded futures contract throughout the world and, as such, the total number of ticks for the continuous time series of the front month contract is equal to $52,099,419$. The trading period for the WTI futures is from 9:00 AM to 2:30 PM, which should provide 60 intraday returns each day (54 intraday returns for the September 2001/January 2007 period where trading began at 10:00 AM). Similarly to the previous asset, we remove days with less than 60 (54) intraday returns, days with more than 15 zero-returns, and days with less than 700 registered ticks. The number of observations is therefore reduced from 2,140 days to 2,081 days when all these requirements are met. The mean number of trades is equal to 25,035. In contrast to S\&P 500 futures, this figure is very different before and after mid-2006, which is mainly due to the launch of electronic trading. ${ }^{24}$

Table A.1: Futures data.

\begin{tabular}{lllll}
\hline \hline \multirow{2}{*}{ Futures contract } & & & & \\
& Exchange & Sample & Trading Hours & Nb of days \\
S\&P 500 & & & & \\
10-year U.S. Treasury Bond & CME & $04 / 21 / 1982-08 / 16 / 2013$ & $8: 30$ a.m.- $3: 15$ p.m. (CT) & 7,662 \\
\$ CAD & CME-CBOT & $01 / 03 / 1989-08 / 16 / 2013$ & $7: 20$ a.m.-2:00 p.m. (CT) & 5,713 \\
WTI Crude Oil & $07 / 21 / 1980-08 / 16 / 2013$ & $7: 20$ a.m.-2:00 p.m. (CT) & 6,869 \\
& CME-NYMEX & $01 / 02 / 1987-08 / 16 / 2013$ & $9: 00$ a.m.-2:30 p.m. (CT) & 6,279 \\
\hline
\end{tabular}

\footnotetext{
${ }^{23}$ We do not build our continuous series using a fixed number of days prior to maturity, thus avoiding calendar effects.

${ }^{24}$ We have an average number of 2,214 ticks per day during the September 4, 2001 / August 31, 2006 period vs. 57,054 ticks for the September 1, 2006 / January 15, 2010 period.
} 

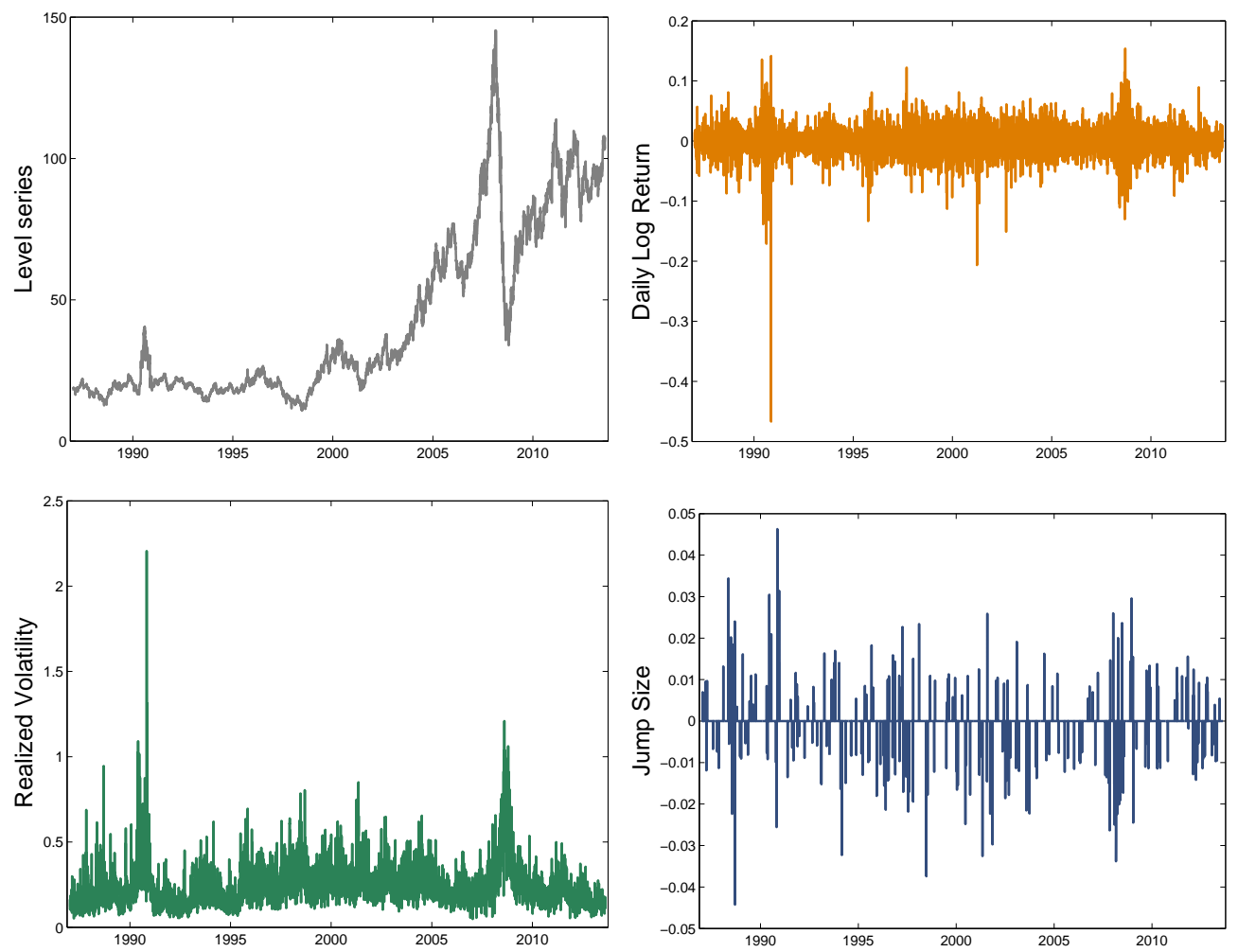

Figure A.1: Detected jumps for the WTI crude oil futures using Lee and Mykland (2008) test associated with intraweek periodicity as in Boudt et al. (2011). The sampling interval for intraday returns is 15 minutes and the threshold for the jump test filtered statistic is $\alpha=0.01$. 

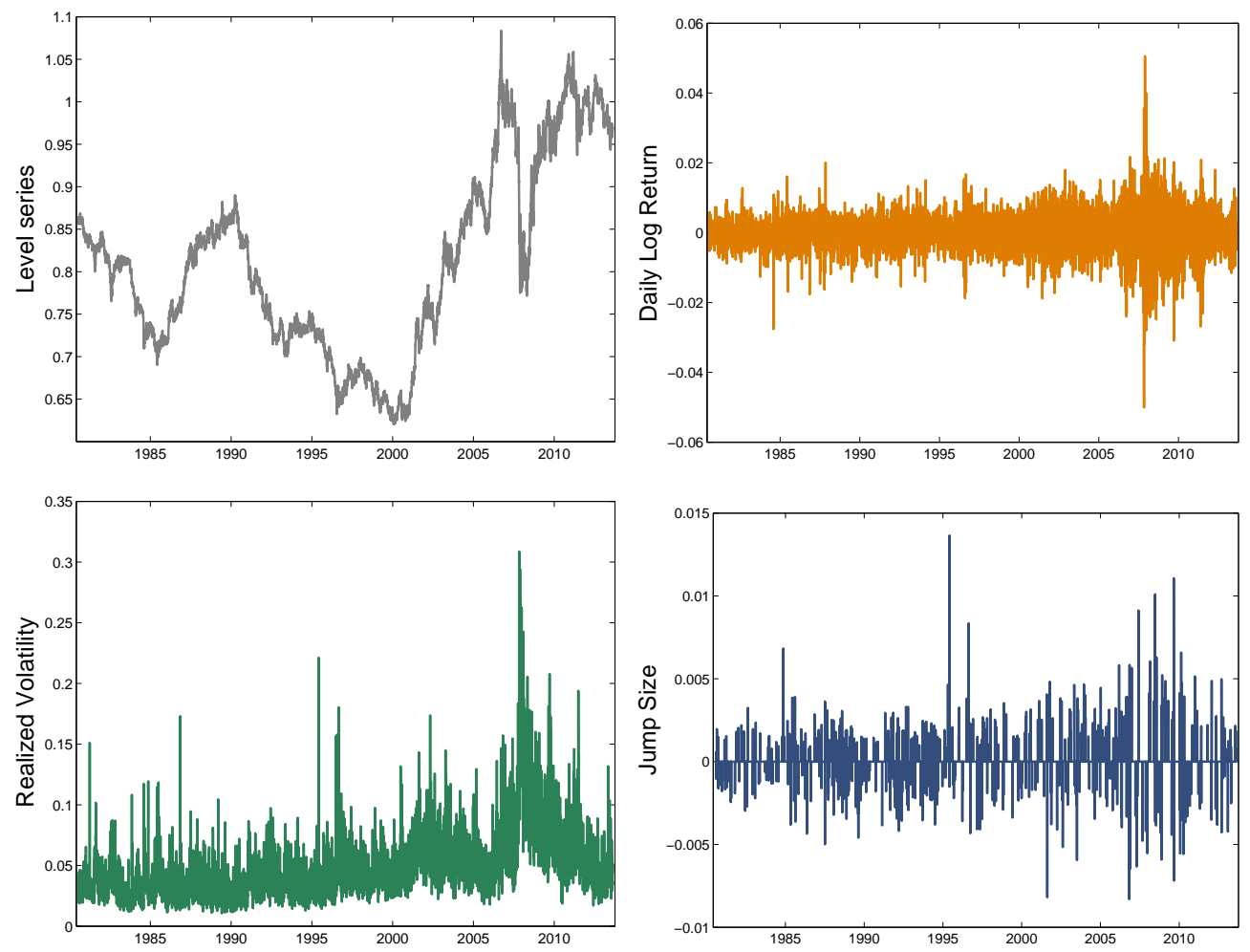

Figure A.2: Detected jumps for USD/CAD futures using Lee and Mykland (2008) test associated with intraweek periodicity as in Boudt et al. (2011). The sampling interval for intraday returns is 15 minutes and the threshold for the jump test filtered statistic is $\alpha=0.01$. 

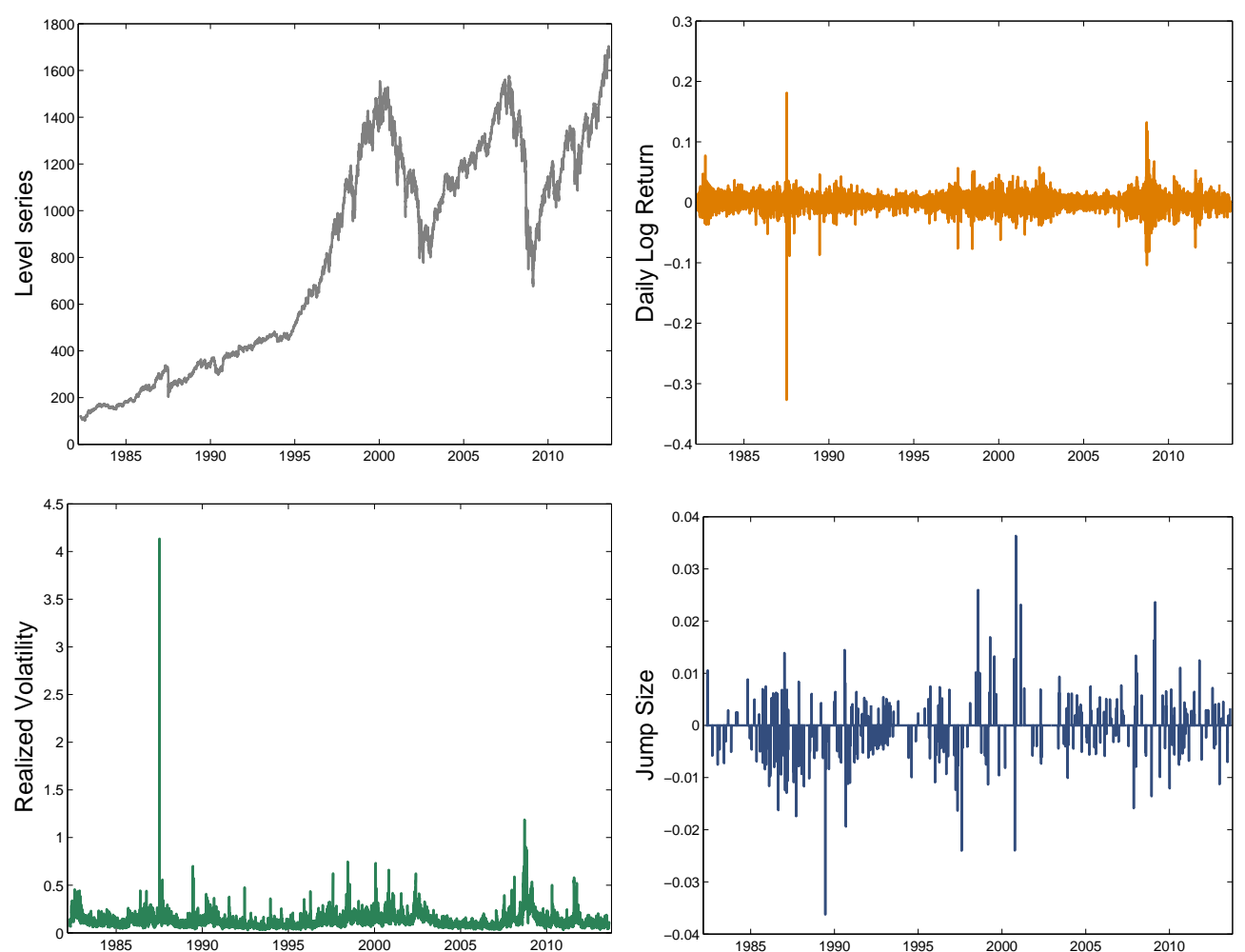

Figure A.3: Detected jumps for the S\&P 500 index futures using Lee and Mykland (2008) test associated with intraweek periodicity as in Boudt et al. (2011). The sampling interval for intraday returns is 15 minutes and the threshold for the jump test filtered statistic is $\alpha=0.01$. 

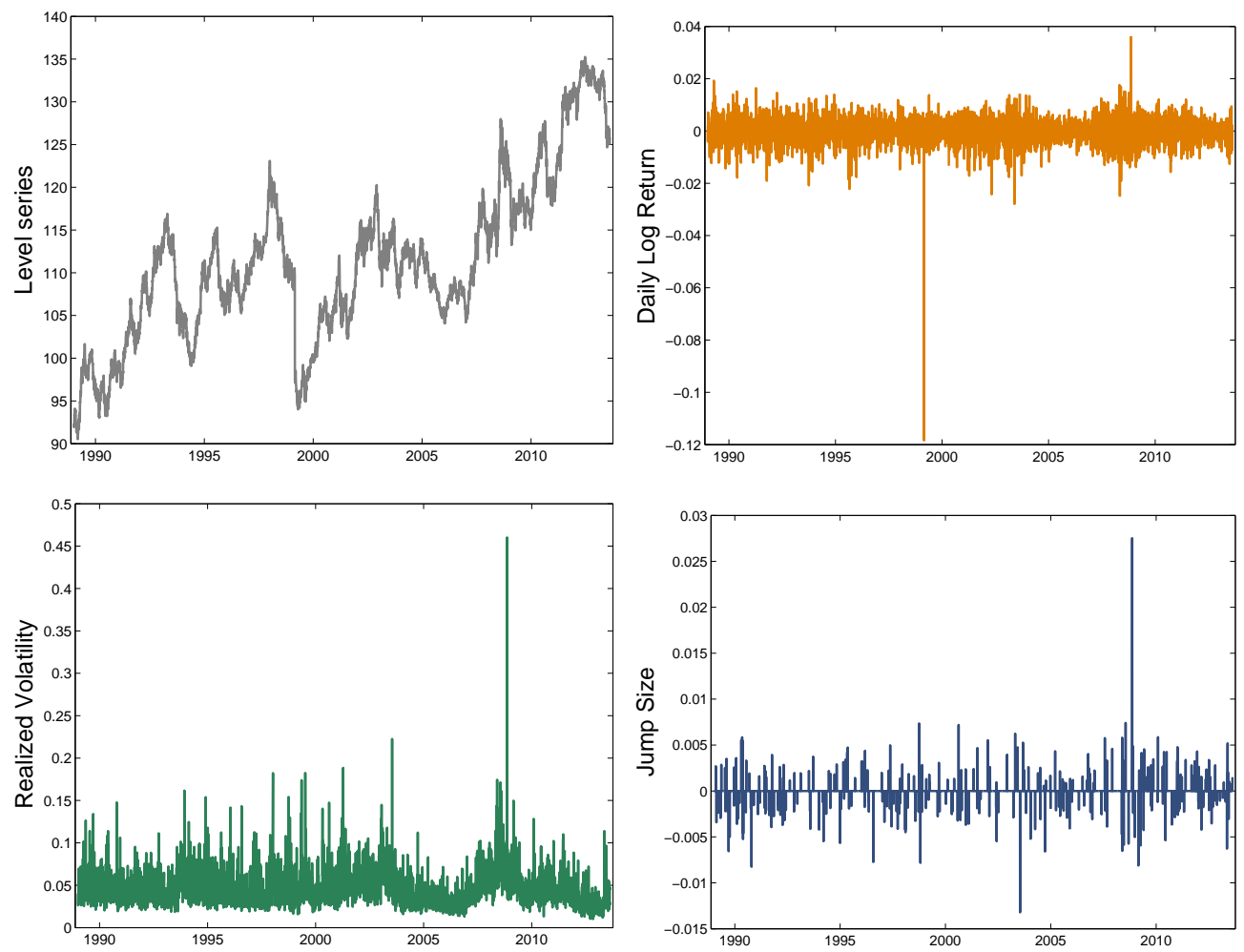

Figure A.4: Detected jumps for the 10-year Treasury Note futures using Lee and Mykland (2008) test associated with intraweek periodicity as in Boudt et al. (2011). The sampling interval for intraday returns is 15 minutes and the threshold for the jump test filtered statistic is $\alpha=0.01$. 